\title{
An Analytical Approach to the Determination of Optimal Perturbations in the Eady Model
}

\author{
MAXIM V. KALASHNIK \\ A. M. Obukhov Institute of Atmospheric Physics, Russian Academy of Sciences, Moscow, and \\ Institute of Experimental Meteorology, Scientific and Production Association "Typhoon," Obninsk, Russia \\ OTTO CHKHETIANI \\ A. M. Obukhov Institute of Atmospheric Physics, Russian Academy of Sciences, Moscow, Russia
}

(Manuscript received 21 March 2018, in final form 29 May 2018)

\begin{abstract}
Within the framework of the baroclinic instability Eady model, an analytical approach to the determination of optimal perturbations with a maximum of the energy growth rate or the ratio of the final and initial energies is considered. This approach is based on the energy balance equation and explicit expressions for the energy functionals resulting from the perturbation representation by means of the superposition of the edge Rossby waves (ERWs). The corresponding expressions are functions of the parameters of the initial perturbation, and the determination of optimal parameters reduces to the study of these functions on an extremum. For perturbations with zero potential vorticity (PV), the amplitudes of the initial buoyancy distributions at the boundaries of the atmospheric layer and the phase shift between these distributions serve as parameters. Analytical formulas are obtained for the optimal phase shift and the maximum of the energy ratio, which determine their dependence on the wavenumber and optimization time. It is also shown that the optimal perturbations always have equal boundary amplitudes. The parameters of the optimal perturbations are compared with the parameters of the growing normal modes. It is established that there exists only one exponentially growing normal mode, which is the optimal perturbation. Along with the instability, the ERWs can be excited by their interaction with the initial vortex perturbations $(P V \neq 0)$. The optimal regime of ERWs excitation by the initial singular distribution of PV is investigated.
\end{abstract}

\section{Introduction}

A zonal flow with a constant vertical shear in the atmospheric layer of finite height is considered in the Eady classical model of baroclinic instability (Eady 1949). The flow stability is studied in the context of a linearized form of the potential vorticity (PV) transport equation in the quasigeostrophic approximation. The wave solutions of this equation are represented by two normal modes (modes of the discrete spectrum with $\mathrm{PV}=0)$ and singular modes of the continuous spectrum $(\mathrm{PV} \neq 0)$. In the long-wavelength range of wavenumbers, one of the normal modes exponentially (in time) grows; the second damps. The fact that the parameters of the growing normal mode are in good agreement with those of atmospheric vortices in the midlatitudes has given impetus to intensive

\footnotetext{
Corresponding author: Otto G. Chkhetiani, ochkheti@gmail.com
}

studies of the Eady model. A rather incomplete list of publications is presented in monographs and reviews (Lindzen 1990; Pierrehumbert and Swanson 1995; Cushman-Roisin and Beckers 2011; Mak 2011; McWilliams 2011).

There are two priority directions in current studies of the Eady model. The first one concerns the description of baroclinic instability in terms of the interaction of edge Rossby waves (ERWs). These waves, which are also often called counterpropagating Rossby waves (CRWs), are shear-flow perturbations localized at both upper and lower boundaries on the Rossby height scale. If the distance between the layer boundaries is on the order of this scale, two ERWs efficiently interact, which results in the development of instability. A system of dynamical equations that describe the interaction process was first formulated by Davies and Bishop (1994) and analyzed by Heifetz et al. (2004a,b) and Heifetz and Methven (2005). 
It is important to emphasize that the interaction of ERWs is described in the context of a problem with initial conditions for perturbations with zero potential vorticity. The statement of this problem is reduced to specifying initial buoyancy distributions at the layer boundaries. The main result of the abovementioned works is that they showed a key role of phase shifts between boundary buoyancy distributions in the development of instability.

The other important direction of research, to which this work belongs, is associated with the concept of optimal perturbations or singular vectors. If $E(t)$ is the total (integral) perturbation energy, one can determine the energy growth rate $\gamma=E^{-1} d E / d t$ (at initial time) or the energy ratio $F=E(t) / E(0)$ (at fixed time $t$ ). Optimal perturbations imply initial perturbations, for which these characteristics (functionals) take on maximum values. Farrell $(1982,1988,1989)$ began to study the problem of optimal perturbations as applied to a wide class of problems in the theory of hydrodynamic stability. In particular, he showed that, in a general case, an exponentially growing normal mode is not an optimal perturbation - the growth rate of the superposition of both growing and decaying modes may be higher than that of the growing mode alone. A mathematical reason for such a behavior, which is associated with the absence of the normality of differential operators in stability theory problems and, as a consequence, the orthogonality of their eigenfunctions, was also found (Farrell and Ioannou 1996). A number of mathematical aspects of the problem were discussed by Trefethen et al. (1993), Schmid and Henningson (2012), Leutbecher (2007), and Diaconescu and Laprise (2012).

In existing works on finding optimal perturbations in the Eady model, as a rule, a sufficiently complex computational problem of finding a conditional extremum of the total-energy functional (or a number of other functionals-enstrophy, potential energy, etc.) is solved. The algorithm of solving this problem uses a vertical finite-difference approximation of the problem's dynamical operator followed by finding a singular decomposition of its matrix (Fischer 1998; Borges and Hartmann 1992; Mukougawa and Ikeda 1994; Morgan 2001; Morgan and Chen 2002; de Vries and Opsteegh 2007; de Vries et al. 2009). For perturbations with $P V=0$, the analytic form of the SVD decomposition was obtained by Fischer (1998). In Faulwetter (2006) were found the parameters of optimal perturbations with a maximum instantaneous energy growth rate. The content of the last two papers practically exhausts the analytical results on finding the optimal perturbations in the Eady model.

In this paper, we consider a direct analytic approach to the determination of optimal perturbations that does not require the use of the technique of singular decomposition of the operator matrix. This approach uses explicit expressions for the energy functionals, which follow from the representation of the perturbations by the sum of the ERWs. The corresponding expressions are functions of the parameters of the initial perturbation, and the determination of the optimal parameters reduces to the study of these functions on an extremum. For perturbations with zero potential vorticity, the amplitudes of the initial buoyancy distributions at the boundaries of the atmospheric layer and the phase shift between these distributions serve as parameters. In our work, for the first time, analytical formulas are obtained for the optimal phase shift and the maximum of the energy ratio, which determine their dependence on the wavenumber and optimization time. It is also shown that the optimal perturbations always have equal boundary amplitudes. The parameters of the optimal perturbations are compared with the parameters of the growing normal modes. It is established that there exists only one exponentially increasing normal mode, which is the optimal perturbation. The wavenumber of this mode is different from the wavenumber of the normal mode with the growth rate maximum.

It should be emphasized that, unlike Faulwetter (2006), our consideration includes perturbations with a maximum energy ratio. When finding perturbations with a maximum energy growth rate, we use the energy balance equation, which allows us to give a physical interpretation of the baroclinic instability. We also determine the parameters of the optimal perturbations in different norms.

The development of perturbations with $\mathrm{PV}=0$ (superposition of normal modes or ERWs) is initiated by the initial buoyancy distributions at the boundaries. The excitation of ERWs can also be caused by their interaction with vortex perturbations $(\mathrm{PV} \neq 0)$. Various aspects of this interaction (including resonance interaction) were considered by Farrell (1984), Thorncroft and Hoskins (1990), Chang (1992), Jenkner and Ehrendorfer (2006), de Vries and Opsteegh (2007), and Kalashnik (2015). The case of the singular distribution of $\mathrm{PV}$, to which the singular wave mode corresponds, or in the terminology of Heifetz and Methven (2005), the kernel Rossby wave (KRW), is more fully investigated. The propagation of KRW leads to the excitation of two ERWs, even if they were absent at the initial time. A system of equations describing the interaction of three waves was first formulated by Dirren and Davies (2004) and subsequently analyzed by de Vries et al. (2009). Using this system and the energy balance equation for the perturbations, we investigated the optimal regime of the ERWs excitation with a maximum of the energy growth rate ERWs at the initial instant of time. It is 
shown that this regime is realized at the optimal phase shift of two ERWs, which depends on the wavenumber and amplitude of the KRW. The asymptotic expressions for this shift and the corresponding maximum of the energy growth rate are obtained.

The main new results of the paper, therefore, consist in obtaining analytic expressions for the parameters of optimal perturbations with $\mathrm{PV}=0$ and describing the regime of optimal excitation of these perturbations by the initial singular distribution of PV.

The article consists of four sections and is organized as follows. In the second section, we consider a problem with initial data for perturbations with zero PV. Two representations of its solution are presented-in the form of the superpositions of normal modes and ERWs. Using these representations, the parameters of optimal perturbations with $\mathrm{PV}=0$ are determined in the third section. In this case, perturbations with a maximum of the energy growth rate and a maximum of the energy ratio are separately considered. The dependence of the parameters of optimal perturbations on the choice of norms is also investigated. In the fourth section, the solution of the general initial problem for perturbations is represented by the sum of the wave and vortex components. The problem of optimal excitation of the wave component (ERWs) by the singular PV distribution is formulated and solved. Frequency interpretation of the effect of resonance excitation of neutral ERWs is given.

\section{The Eady model and an initial-value problem for perturbations with zero PV}

\section{a. Representation of the problem solution as a sum of normal modes}

A zonal flow (along the horizontal axis $x$ ) with the velocity distribution $U(z)=\Lambda z(\Lambda>0$ is a vertical shear) in an atmospheric layer with thickness $H$ and constant values of buoyancy frequency $N$ and inertial frequency $f$ is considered in the Eady classical model. In a quasigeostrophic approximation, the behavior of two-dimensional flow perturbations is described by the PV transport equation (Pedlosky 1987):

$$
(\partial / \partial t+\Lambda z \partial / \partial x) q=0, \quad q=\psi_{x x}+(f / N)^{2} \psi_{z z},
$$

with the boundary conditions

$$
z=0, H: \quad(\partial / \partial t+\Lambda z \partial / \partial x) \psi_{z}-\Lambda \psi_{x}=0 .
$$

Here, $\psi=p^{\prime} / f$ is a geostrophic streamfunction, and $p^{\prime}$ is the pressure perturbation normalized to mean density $\rho_{0}$. The horizontal wind velocity components $u, v$ and buoyancy $\sigma$ are related to the streamfunction by the relations $u=-\psi_{y}, v=\psi_{x}$, and $\sigma=f \psi_{z}$. In the Boussinesq approximation, $\sigma=-g \rho / \rho_{0}$, where $\rho$ is the density deviation from the equilibrium hydrostatic distribution. Boundary conditions (2.2) are the buoyancy equation written for solid layer boundaries. Note that, along with conventional symbols, here and below, the partial derivatives are denoted by inferior letters.

The perturbations with PV $q=0$ satisfy (2.1). In dimensionless variables, the dynamics of such perturbations is described by the solution of the Laplace equation $\psi_{x x}+$ $\psi_{z z}=0$ with the nonstationary boundary conditions:

$$
z=0,1: \quad(\partial / \partial t+z \partial / \partial x) \psi_{z}-\psi_{x}=0 .
$$

Here, $L_{R}=N H / f$ (baroclinic Rossby radius of deformation), $H$, and $N / \Lambda f$ are taken as the $x, z$, and $t$ scales, respectively. Note that, for the tropospheric characteristic parameters $H=10 \mathrm{~km}, N=10^{-2} \mathrm{~s}^{-1}, f=10^{-4} \mathrm{~s}^{-1}$, and the vertical shear $\Lambda=10^{-3} \mathrm{~s}^{-1}, L_{R}=1000 \mathrm{~km}$ and $T=$ $N / \Lambda f=27.8 \mathrm{~h}$ (approximately 1 day).

Below, we shall consider the case of spatially periodic perturbations. One can write the general solution of Laplace equation for such perturbations in the form

$\psi=\left\{A(t) \frac{\cosh (k z)}{k \sinh (k)}-B(t) \frac{\cosh [k(z-1)]}{k \sinh (k)}\right\} e^{i k x}$,

where $A(t), B(t)$ are the complex amplitudes. It follows from (2.4) that

$$
\begin{aligned}
& \left.\psi_{z}\right|_{z=0}=B(t) e^{i k x}, \\
& \left.\psi_{z}\right|_{z=1}=A(t) e^{i k x} .
\end{aligned}
$$

Substituting (2.4) into conditions (2.3) with consideration for (2.5) yields the system of linear ordinary differential equations in order to find amplitudes:

$$
\begin{aligned}
i \frac{d \mathbf{r}}{d t} & =\mathbf{S r}, \quad \mathbf{r}(t)=\left[\begin{array}{c}
A(t) \\
B(t)
\end{array}\right], \\
\mathbf{S} & =\left[\begin{array}{cc}
k-\operatorname{coth}(k) & \sinh ^{-1}(k) \\
-\sinh ^{-1}(k) & \operatorname{coth}(k)
\end{array}\right] .
\end{aligned}
$$

The solution of system (2.6) is represented in the form $\mathbf{r}(t)=C_{1} e^{-i \omega_{1} t} \mathbf{h}_{1}+C_{2} e^{-i \omega_{2} t} \mathbf{h}_{2}$, where $C_{1,2}$ are the arbitrary constants, $\mathbf{h}_{1,2}$ are the eigenvectors of the $\mathbf{S}$ system matrix, and $\omega_{1,2}$ are its eigenvalues,

$$
\begin{aligned}
\omega_{1,2} & =k / 2 \pm \sinh ^{-1}(k) \sqrt{\alpha^{2}-1}, \\
\alpha & =\alpha(k)=\cosh (k)-0.5 k \sinh (k) .
\end{aligned}
$$

In view of the expression for the eigenvectors, one can write the solution in the component-wise form 
$[A(t), B(t)]=C_{1}(1 \pm \delta) e^{-i \omega_{1} t}-C_{2}(1 \mp \delta) e^{-i \omega_{2} t}$,

where $\delta^{2}=(\alpha-1) /(\alpha+1)$. Substituting (2.8) into (2.4), we obtain the expression for the streamfunction of perturbations with zero PV:

$$
\begin{aligned}
\psi & =C_{1} F_{1}(z) e^{i\left(k x-\omega_{1} t\right)}-C_{2} F_{2}(z) e^{i\left(k x-\omega_{2} t\right)}, \\
F_{1,2}(z) & =\frac{\sinh [k(z-0.5)]}{k \cosh (0.5 k)} \pm \delta \frac{\cosh [k(z-0.5)]}{k \sinh (0.5 k)} .
\end{aligned}
$$

It is important to emphasize that the arbitrary constant $C_{1,2}$ in (2.9) are unambiguously expressed through initial amplitudes $A(0), B(0)$.

In representation (2.9) of the initial-value problem solution, each summand describes a normal mode with frequency $\omega_{1}\left(\omega_{2}\right)$. The temporal behavior of normal modes is determined by frequency values. It follows from (2.7) that the frequencies $\omega_{1,2}$ are complex if $-1<$ $\alpha(k)<1$ or (which is equivalent) $0<k<k_{b}$, where $k_{b}=$ 2.399 (boundary value) is the root of the equation, $\operatorname{coth}(k / 2)=k / 2[\alpha(k)=-1]$. For $k$ values within the indicated interval, the flow is unstable-in parallel with a damping mode, there is an exponentially growing normal mode. The growth increment of this mode

$$
s(k)=\sinh ^{-1}(k)\left(1-\alpha^{2}\right)^{1 / 2} \equiv\left[k \operatorname{coth}(k)-(k / 2)^{2}-1\right]^{1 / 2}
$$

vanishes at the extremities of the interval and reaches maximum $s=s_{m}=0.310$ at $k=k_{m}=1.606$. For the above-given values of the tropospheric parameters, the wavelength $\lambda_{m}=3.59 L_{R} \sim 3600 \mathrm{~km}$ (one-quarter of wavelength is the cyclone scale) and the $e$-folding time (approximately 4 days) correspond to the most unstable mode $\left(k=k_{m}\right)$ in dimensional variables. These estimates supplemented by studies of the unstable $\mathrm{m} k=k_{m}$ ode structure are the famous results first obtained by Eady (1949).

Note that the above analysis of stability slightly differs from a conventional analysis based on the method of normal modes, when particular wave solutions of system (2.1) and (2.2) are found at once. The application of this method to the general three-dimensional problem (Pedlosky 1987) shows that two-dimensional perturbations have maximum growth rate. Therefore, we confine ourselves to considering only such perturbations.

\section{b. Representation of the initial-value problem solution as a sum of ERWs}

Unlike (2.9), the representation of the initial-value problem solution, which does not call for the isolation of a real part and allows a physical interpretation, was given by Davies and Bishop (1994). One can obtain the corresponding representation from (2.4) if

$$
A(t)=a(t) e^{i \theta_{2}(t)}, \quad B(t)=b(t) e^{i \theta_{1}(t)},
$$

where $a(t), b(t)$ are the real amplitudes and $\theta_{1,2}(t)$ are the corresponding phases. In view of (2.11), for the streamfunction and buoyancy distribution, one can obtain from (2.4) the representation in the real-valued form

$$
\begin{aligned}
\psi= & a(t) \frac{\cosh (k z)}{k \sinh (k)} \cos \left[k x+\theta_{2}(t)\right]-b(t) \frac{\cosh [k(z-1)]}{k \sinh (k)} \\
& \times \cos \left[k x+\theta_{1}(t)\right] \\
\psi_{z}= & a(t) \frac{\sinh (k z)}{\sinh (k)} \cos \left[k x+\theta_{2}(t)\right]-b(t) \frac{\sinh [k(z-1)]}{\sinh (k)} \\
& \times \cos \left[k x+\theta_{1}(t)\right]
\end{aligned}
$$

The initial buoyancy distributions at the boundaries are described by the expressions

$$
\begin{aligned}
& \left.\psi_{z}\right|_{z=1}=a(0) \cos \left[k x+\theta_{2}(0)\right] \quad \text { and } \\
& \left.\psi_{z}\right|_{z=0}=b(0) \cos \left[k x+\theta_{1}(0)\right] .
\end{aligned}
$$

In representations (2.12) and (2.13), the summands proportional to $a(t), b(t)$ describe ERWs-the waves localized at the upper (lower) boundary, respectively. As is indicated above, it is conventional to call a pair of such waves the counterpropagating Rossby waves. The system of dynamical equations, which describes the interaction of the wave pair, can be obtained by substituting (2.11) into system (2.6) after the isolation of both real and imaginary parts:

$$
\begin{aligned}
& d a / d t=r b \sin (\theta), \quad d b / d t=r a \sin (\theta), \\
& d \theta / d t=-2 r\left[\alpha-0.5\left(m+m^{-1}\right) \cos (\theta)\right], \quad m=b / a,
\end{aligned}
$$

$d \theta_{+} / d t=-\left[k+r\left(m-m^{-1}\right) \cos (\theta)\right]$.

Here, $\theta=\theta_{1}-\theta_{2}, \theta_{+}=\theta_{1}+\theta_{2}$, and for short, $r=$ $\sinh ^{-1}(k)$. Note that (2.14a) and (2.14b) form a closed system for finding $a, b, \theta$. This system was first formulated by Davies and Bishop (1994).

Although system (2.14) is nonlinear, unlike system (2.6), it illustrates the key role of the phase shift $\theta=\theta_{1}-\theta_{2}$ for the development of initial perturbations. In fact, according to $(2.14 \mathrm{a})$, the amplitudes increase if the phase shift $0<$ $\theta<\pi[\sin (\theta)>0]$. Then, at $a=b$ and $\theta=\theta_{n}$, where

$$
\cos \left(\theta_{n}\right)=\alpha(k) \equiv \cosh (k)-0.5 k \sinh (k),
$$

System (2.14a) and (2.14b) have the exact solution $a(t)=a(0) e^{s t}$. This solution corresponds to the normal 
mode with growth increment (2.10). For this mode, $\theta=\theta_{n}$ is the fixed point of (2.14b) at $a=b$. This point exists only for $-1<\alpha(k)<1$ (i.e., for the wavenumbers within the earlier obtained interval $0<k<k_{b}$ ).

Equation (2.15) determines the dependence of the phase shift $0<\theta_{n}<\pi$ on the wavenumber of the normal growing mode. For $k=k_{s}=2.065$, where $k_{s}$ is the root of the equation $\operatorname{coth}(k)=0.5 k[\alpha(k)=0], \theta_{n}=\pi / 2$. In a certain sense, $k=k_{s}$ is boundary-at $k<k_{s}\left(k>k_{s}\right)$, the $\theta_{n}$ angle is acute (obtuse). We should emphasize that there is no information on phase shift in representation (2.9) of the initial-value problem solution.

Davies and Bishop (1994) analyzed a number of other exact solutions of system (2.14) and indicated its important first integrals $I_{1,2}=$ const,

$I_{1}=a^{2}-b^{2}, \quad I_{2}=0.5\left(a^{2}+b^{2}\right)-\alpha^{-1} a b \cos (\theta)$.

The Hamiltonian formulation of the system and a number of physical interpretations (in the context of more complicated models) were later given by Heifetz et al. (2004a,b).

\section{Optimal perturbations with zero PV}

\section{a. Energy balance equation and optimal initial perturbations}

The energy balance equation, which follows from (2.1) and (2.2), plays an important role in our consideration of the problem of optimal perturbations. Introducing both horizontal and volume averaging operations

$$
\bar{\varphi}^{x}=\lim _{L \rightarrow \infty} \frac{1}{2 L} \int_{-L}^{L} \varphi d x \quad \text { and } \quad\langle\varphi\rangle \equiv \int_{0}^{1} \bar{\varphi}^{x} d z,
$$

we determine the energy density and the total perturbation energy from the expressions (dimensionless form)

$$
\varepsilon=\frac{1}{2}\left(\psi_{x}^{2}+\psi_{z}^{2}\right), \quad E=\langle\varepsilon\rangle \equiv \int_{0}^{1} \bar{\varepsilon}^{x} d z .
$$

The differentiation of $\varepsilon$ with respect to time results in the identity

$$
\frac{\partial \varepsilon}{\partial t}=\frac{\partial}{\partial x}\left(\psi \psi_{x t}\right)+\frac{\partial}{\partial z}\left(\psi \psi_{z t}\right)-\psi \frac{\partial}{\partial t} q,
$$

where $q=\psi_{x x}+\psi_{z z}$. For perturbations with $q=0$, the horizontal averaging of (3.2) yields

$$
\frac{\partial \bar{\varepsilon}^{x}}{\partial t}=\frac{\partial}{\partial z}\left(\overline{\psi \psi}_{z t}^{x}\right)
$$

Integrating (3.3) with the use of boundary conditions (2.3), we obtain the energy balance equation for perturbations with zero PV:

$$
\frac{d E}{d t}=\left.{\overline{\psi_{x}} \psi_{z}}^{x}\right|_{z=1}
$$

According to this equation, total-energy variations are determined by a meridional buoyancy flux at the upper boundary.

Using representation (2.12) of the initial-value problem solution, direct integration, and standard rules of averaging periodic functions yields the explicit expressions for the total energy and its derivative:

$$
\begin{aligned}
\frac{d E}{d t} & =\left.\bar{\psi}_{x} \psi_{z}{ }^{x}\right|_{z=1}=\frac{1}{2} \sinh ^{-1}(k) a(t) b(t) \sin [\theta(t)], \\
E(t) & =\frac{1}{2}\left\langle\psi_{x}^{2}+\psi_{z}^{2}\right\rangle \\
& =\frac{1}{4} \frac{\operatorname{coth}(k)}{k}\left\{\begin{array}{l}
a^{2}(t)+b^{2}(t) \\
-2 a(t) b(t) \cosh ^{-1}(k) \cos [\theta(t)]
\end{array}\right\} .
\end{aligned}
$$

The important physical result follows directly from (3.5) - the total energy increases if the phase shift is $0<$ $\theta<\pi$. Thus, this result may be obtained without using system (2.14) for the description of the interaction of ERWs. Note that perturbations can extract energy from shear flow only at the indicated phase shift.

Expressions (3.5) and (3.6) make it possible to obtain a simple solution of the problem of initial optimal perturbations-perturbations with a maximum energy growth rate at initial time. The expression for the energy growth rate, which follows from (3.5) and (3.6),

$$
\gamma=\frac{1}{E} \frac{d E}{d t}=\frac{2 k}{\cosh (k)} \times \frac{a b \sin (\theta)}{a^{2}+b^{2}-2 a b \cosh ^{-1}(k) \cos (\theta)},
$$

depends (at fixed $k$ ) on the three variables $a, b, \theta$-initial amplitude and phase shift values. The values of the variables, at which the energy growth rate (3.7) takes on maximum value $\gamma=\gamma_{\mathrm{opt}}(k)$, correspond to optimal perturbations. Below, we will consider $k$ values within the interval $0<k<k_{b}$ of the existence of growing normal modes. Note that, in view of (2.15) and (3.7), the energy growth rate of an unstable normal-mode $\gamma=\gamma_{n}(k)=2 s(k)$ amounts to the doubled increment of growth. As is shown below, the value of $\gamma_{n}(k)$ is not optimal.

Since $\gamma$ is the homogeneous function of amplitudes, it reaches its maximum at $a=b$. To make certain that this is the case, one can directly assume that $a=\rho \cos (\varphi)$ and $b=\rho \sin (\varphi)$. It is easy to check that, at fixed $k, \theta$ values, the maximum of (3.7) is reached at $\sin (2 \varphi)=1$. Hence, $\varphi=\pi / 4$ and $a=b$. 


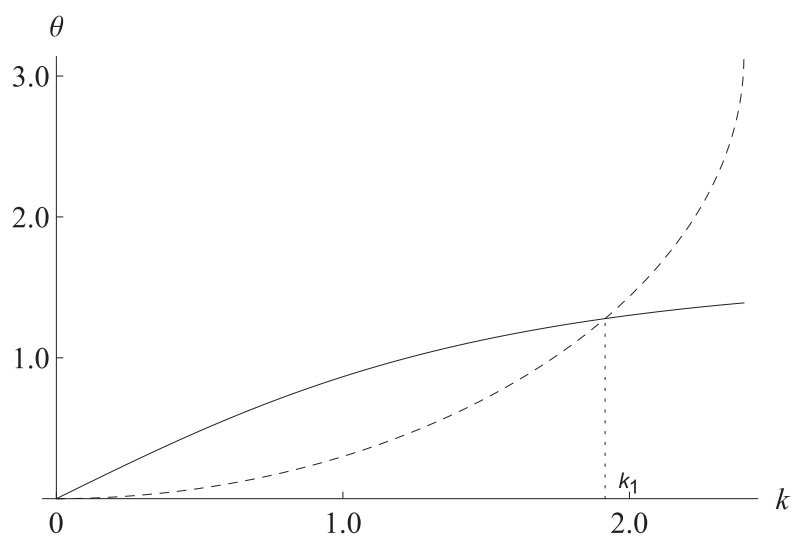

FIG. 1. Dependences of $\theta_{\text {opt }}$ (solid line) and $\theta_{n}$ (dashed line) on the wavenumber $k$ within the interval $0<k<k_{b}$. The value of $k_{1}$, for which a normal mode is an optimal perturbation, is marked.

At $a=b$ and a fixed wavenumber, the energy growth rate

$$
\gamma=\gamma(k, \theta)=\frac{k \sin (\theta)}{\cosh (k)-\cos (\theta)}
$$

depends only on the initial phase shift. Since $\gamma>0$ within the interval $0<\theta<\pi$ and at its ends vanishes, there is a value of $\theta=\theta_{\text {opt }}$, at which the function $\gamma(k, \theta)$ reaches its maximum. This value corresponds to the initial optimal perturbation. An elementary analysis of (3.8) for an extremum yields

$$
\cos \theta_{\mathrm{opt}}=\cosh ^{-1}(k), \quad \gamma_{\mathrm{opt}}=\gamma\left(k, \theta_{\mathrm{opt}}\right)=k / \sinh (k) .
$$

Equations (3.9) determine the dependences of both phase shift and energy growth rate on the wavenumber of optimal perturbation. Note that, unlike unstable normal modes, for optimal perturbations, the phase shift is $0<\theta_{\text {opt }}<\pi / 2$. As a wavenumber function, the energy growth rate reaches its maximum in the long-wave limit: $\gamma_{\mathrm{opt}} \rightarrow 1$ at $k \rightarrow 0$.

Comparison of (3.9) and (2.15) shows that, in the general case, $\theta \neq \theta_{\text {opt }}$; that is, the exponentially growing normal modes are not optimal perturbations. Figure 1 shows the dependences of $\theta_{\text {opt }}(k)$ and $(k)$ on the wavenumber $k$. Figure 2 shows the graphs of $\gamma_{\text {opt }}(k)$ and $\gamma_{n}(k)=2 s(k)$ dependences and the graph of the ratio $\phi(k)=\gamma_{n}(k) / \gamma_{\text {opt }}(k)$. It follows from these figures that there exists a unique wavenumber value ( $\left.k=k_{1}=1.915\right)$, for which the normal mode is optimal; that is, $\cos \theta_{n}=\cos \theta_{\text {opt }}$ and $\gamma_{\text {opt }}=\gamma_{n}$. The phase shift $\theta_{n}=\theta_{\mathrm{opt}}=73.22^{\circ}$ corresponds to this value (root of the equation $\tanh k=k / 2$ ). At $k<k_{1}\left(k>k_{1}\right)$, $\theta_{n}<\theta_{\text {opt }}\left(\theta_{n}>\theta_{\text {opt }}\right)$. Note that the two graphs of the energy growth rate touch each other at the point $k=k_{1}$. For all $k \neq k_{1}$ values, $\gamma_{\text {opt }}>\gamma_{n}$.

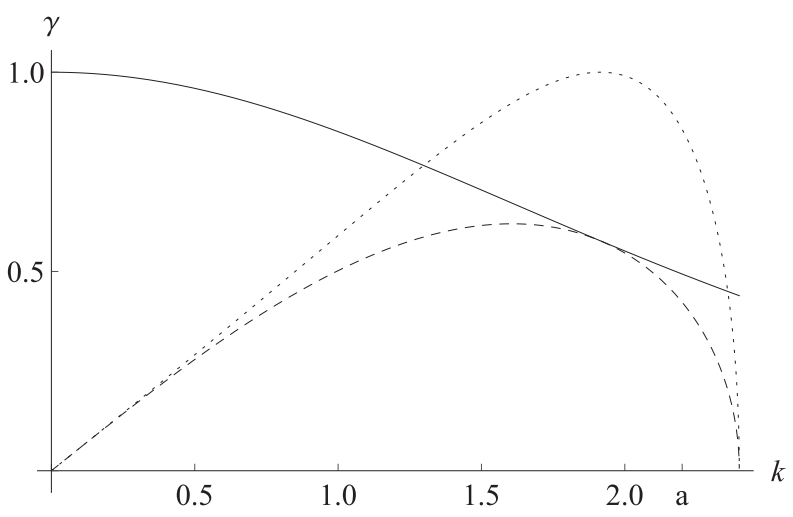

FIG. 2. Dependences of $\gamma_{\text {opt }}$ (solid line), $\gamma_{n}$ (dashed line), and $\varphi=\gamma_{n} / \gamma_{\text {opt }}$ (dotted line) on the wavenumber $k$.

Let us give some numerical estimates for $k=k_{m}=$ 1.606 , which corresponds to the most unstable normal mode. For this value, $\cos \theta_{n}=0.672\left(\theta_{n}=47.8^{\circ}\right)$ and $\cos \theta_{\text {opt }}=0.386\left(\theta_{\text {opt }}=67.3^{\circ}\right)$. The energy growth rate for a normal-mode $\gamma_{n}=0.62$ is $8 \%$ less than that for an optimal perturbation $\gamma_{\mathrm{opt}}=0.672$. The characteristic time of energy growth for a normal-mode $\tau_{n}=1 / \gamma_{n}$ in dimensional variables amounts to $44.9 \mathrm{~h}$. For an optimal perturbation, this time is $\tau_{\mathrm{opt}}=1 / \gamma_{\mathrm{opt}}=41.4 \mathrm{~h}$. Note that a slight difference of $\tau$ values is associated with the chosen value of $k$. It follows from Fig. 2 that, for $k=1$, the energy growth rate for a normal mode is almost 2 times lower than that for an optimal perturbation.

Figures $3 \mathrm{a}$ and $3 \mathrm{c}$ give the grayscale plot of the isolines of the buoyancy field $\sigma=\psi_{z}$ (2.13) for a normal mode and optimal perturbation, respectively, at $k=k_{m}=1.606$ and $\theta_{2}=0, \theta_{1}=\theta$. If the phase shift is positive, the field isolines are inclined along the flow, and this inclination is stronger for an optimal perturbation. Figures $3 \mathrm{~b}$ and $3 \mathrm{~d}$ show the isolines of pressure field (2.12) ( $\psi$ field) at the same value of $k$. Here, the isolines are inclined in the direction opposite to that of the flow. This is because the phase shift between boundary pressure distributions is negative. It can be shown that for an optimal perturbation, this shift is equal to that between buoyancy distributions, which is taken with opposite sign.

Note that, because of a strong difference between horizontal and vertical scales, the phase shift shown in Fig. 3 is conditional. The differences in the phase shifts between an optimal perturbation and a normal mode are more clearly pronounced for the wavenumber $k=2.35$, which is close to the boundary value of $k_{b}$ (Fig. 4).

One more informative characteristic is the phase shift $\vartheta$ between pressure and buoyancy distributions at different levels, in particular, at the ground level. Since, at the lower boundary, a low pressure corresponds to a higher temperature (buoyancy), it is convenient to consider 


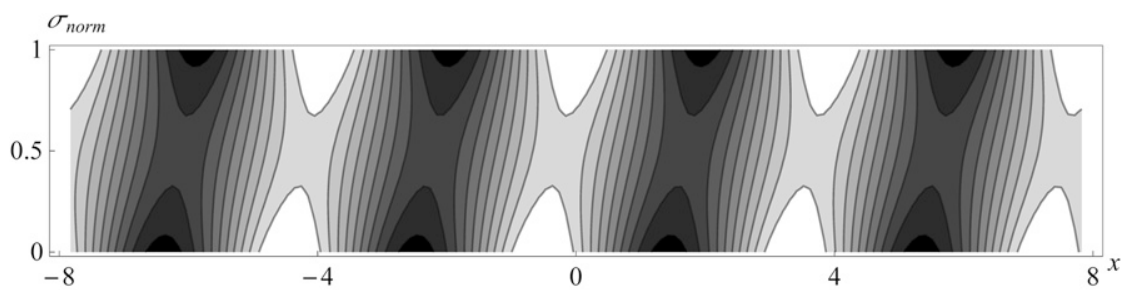

a)
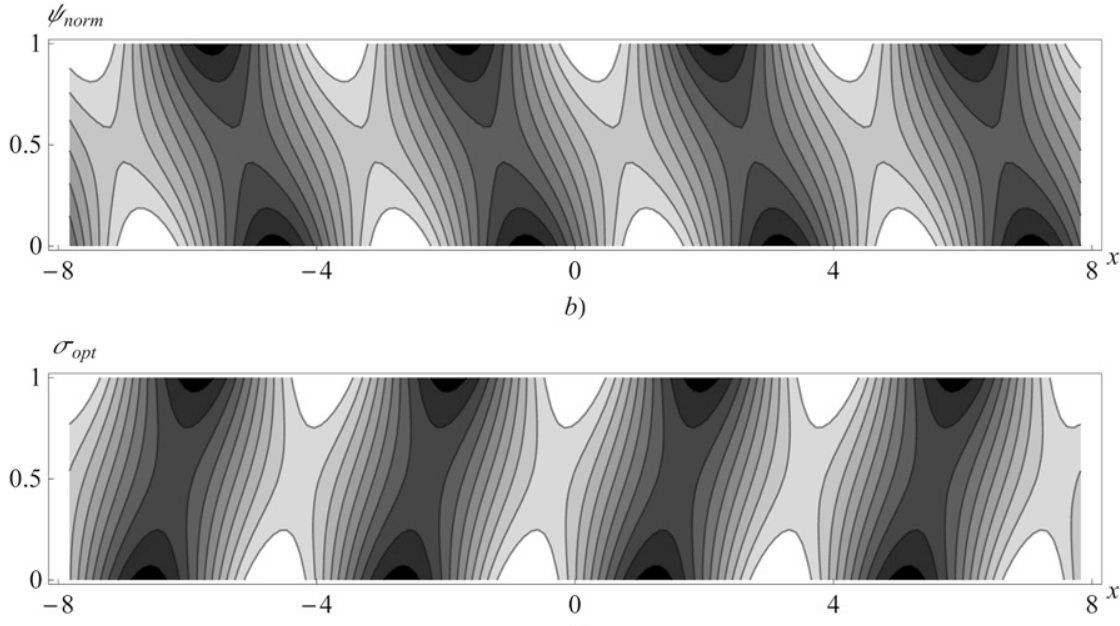

c)

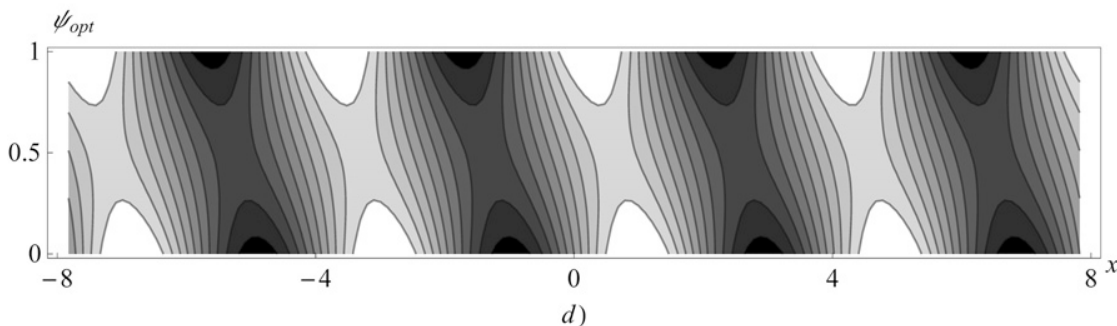

FIG. 3. Grayscale plot of the isolines of both (a),(c) buoyancy and (b),(d) pressure fields for the (a),(b) normal mode and (c),(d) the initial optimal perturbation at $k=1.606$.

the pressure distribution opposite in sign. This phase shift is $\vartheta=(\pi / 2)-\theta_{\mathrm{opt}}=22.7^{\circ}$ for an optimal perturbation with the wavenumber $k=k_{m}$ and $\vartheta=\arctan [2 s(k) / k]=$ $21.1^{\circ}$ for a normal mode. In this case, the maximum of potential temperature is always slightly ahead of pressure trough (minimum).

Concluding this section, we will give the expressions for phase shifts (2.15) and (3.9), which are represented in terms of half-angle tangent:

$$
\begin{aligned}
\tan \left(\theta_{n} / 2\right) & =\delta \equiv\left[\frac{(k / 2)-\tanh (k / 2)}{\operatorname{coth}(k / 2)-(k / 2)}\right]^{1 / 2}, \\
\tan \left(\theta_{\mathrm{opt}} / 2\right) & =\tanh (k / 2) .
\end{aligned}
$$

Here and below, $\delta=[(1-\alpha) /(1+\alpha)]^{1 / 2}$. These expressions will be used in the following section.

\section{b. Optimal perturbations with a maximum ratio between final and initial energies}

Now let us consider a more general problem of optimal perturbations. This problem is formulated as follows-for fixed time $t$, to find an initial perturbation, for which the functional $F=E(t) / E(0)$ takes on its maximum value. Below, the solution of this problem in a class of perturbations with equal initial amplitudes at the boundaries is given. By virtue of the conservation law $I_{1}$ (2.16), the equality of the amplitudes is conserved at an arbitrary time. The class of perturbations under consideration, which includes normal modes, is sufficiently general.

Note that, in view of the expansion

$$
F=\frac{E(t)}{E(0)}=1+\gamma t, \quad \gamma=\left.\frac{1}{E} \frac{d E}{d t}\right|_{t=0} t \ll 1,
$$




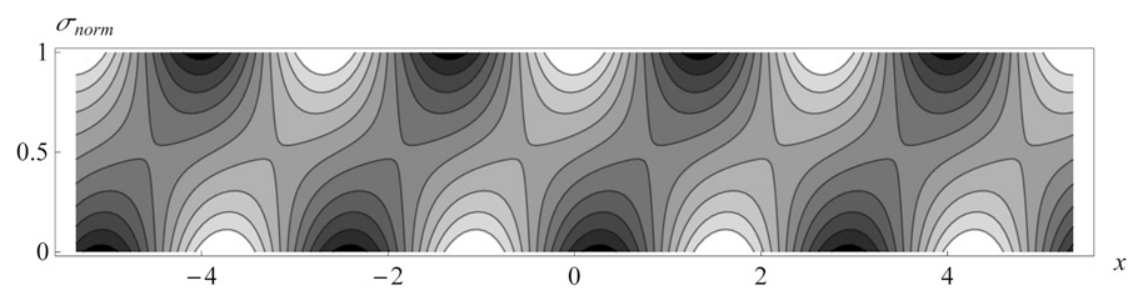

a)

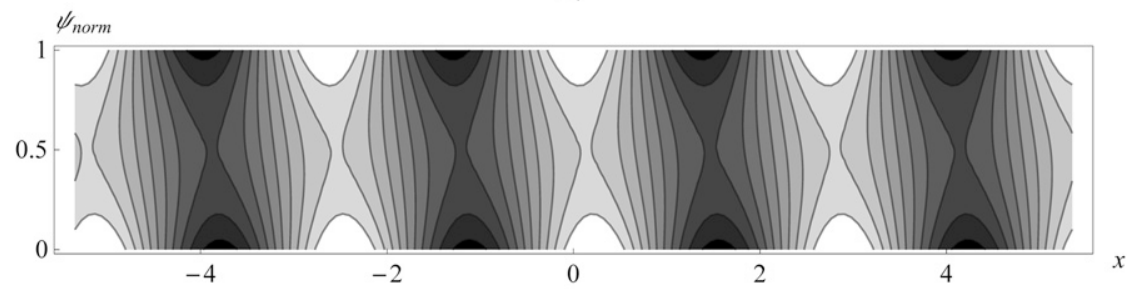

b)

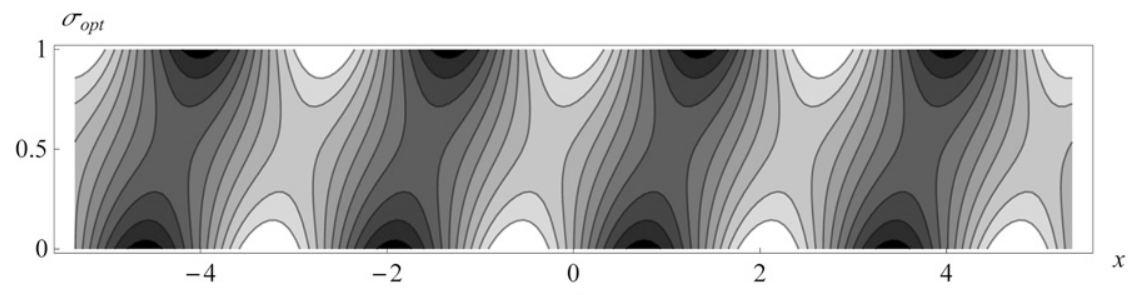

c)

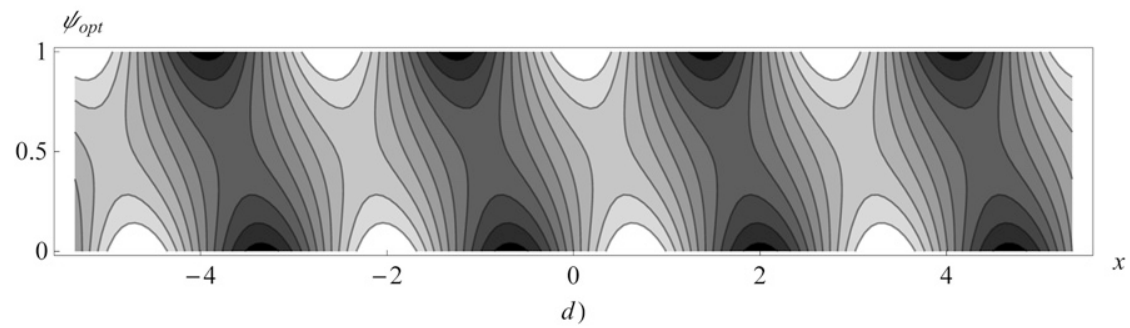

FIG. 4. As in Fig. 3, but for $k=2.35$.

the optimal perturbations found in the previous section yield the solution of the problem for $t \rightarrow 0$. For finite values of $t$, the problem solution calls for the integration of the system of dynamical (2.14).

For perturbations with $a=b$, total energy (3.6)

$$
E(t)=\frac{1}{2} \frac{\operatorname{coth}(k)}{k} a^{2}(t)\left\{1-\cosh ^{-1}(k) \cos [\theta(t)]\right\},
$$

and the energy ratio

$F=\frac{E(t)}{E(0)}=\left[\frac{a^{2}(t)}{a^{2}(0)}\right]\left\{\frac{\cosh (k)-\cos [\theta(t)]}{\cosh (k)-\cos \left(\theta_{0}\right)}\right\}$.

Here, $\theta(0)=\theta_{0}$. Using first integral $I_{2}(2.16)$ yields the equality

$$
\frac{a^{2}(t)}{a^{2}(0)}=\frac{\alpha-\cos \left(\theta_{0}\right)}{\alpha-\cos [\theta(t)]},
$$

which makes it possible to transform (3.12) to the form

$$
F=\frac{E(t)}{E(0)}=\left[\frac{\alpha-\cos \left(\theta_{0}\right)}{\cosh (k)-\cos \left(\theta_{0}\right)}\right]\left\{\frac{\cosh (k)-\cos [\theta(t)]}{\alpha-\cos [\theta(t)]}\right\} .
$$

In (3.13), the phase shift $\theta(t)$ is the solution of the Cauchy problem for $(2.14 b)$ :

$$
d \theta / d t=-2 r[\alpha-\cos (\theta)], \quad \theta(0)=\theta_{0}
$$

which depends on the initial value of $\theta_{0}$ and the wavenumber $k$ as on the parameters: $\theta(t)=\theta\left(t, k, \theta_{0}\right)$. In view of this dependence, the functional $F$ is the function of the three variables $F=F\left(t, k, \theta_{0}\right)$. Determining an optimal perturbation is reduced to finding a maximum of this function at fixed values of the first two arguments. Let us emphasize that $\theta_{0}$ is the initial phase shift. 


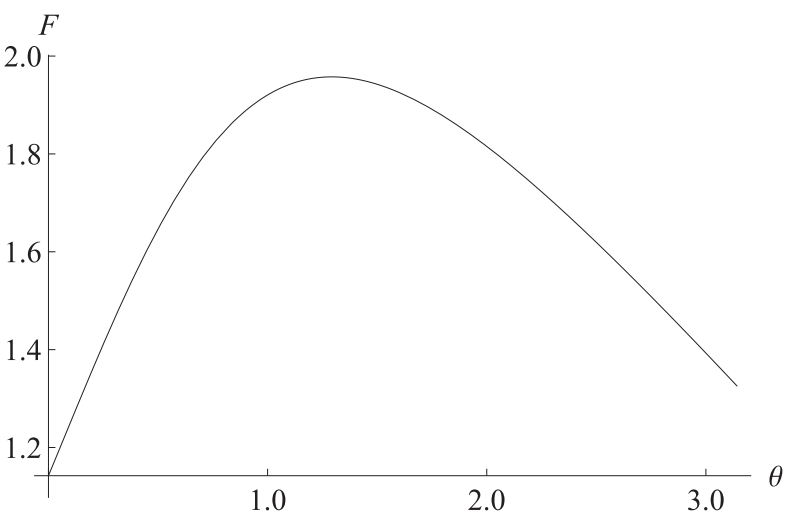

FIG. 5. Dependence of the energy ratio $F$ on the phase shift $\theta$ at $k=1.606$ and $t=1$.

An explicit expression for the function $F=F\left(t, k, \theta_{0}\right)$ may be obtained by integrating (3.14). The standard separation of the variables yields the exact solution $\theta(t)=\theta\left(t, k, \theta_{0}\right)$ in the form of the first integral

$$
\begin{aligned}
Y & =\frac{X+c(t)}{c(t) X+1}, \quad Y=\delta^{-1} \tan [\theta(t) / 2], \\
X & =\delta^{-1} \tan \left(\theta_{0} / 2\right),
\end{aligned}
$$

where $c(t)=\tanh [s(k) t]$. Expressing $\cos \theta(t)$ in (3.13) through the half-angle tangent and using (3.15), after transformations, we obtain the expression

$$
\begin{aligned}
F & =\frac{1}{1-c^{2}(t)} \frac{[X+c(t)]^{2}+n[c(t) X+1]^{2}}{X^{2}+n}, \\
X & =\delta^{-1} \tan \left(\theta_{0} / 2\right),
\end{aligned}
$$

which determines the function $F=F\left(t, k, \theta_{0}\right)$ or, which is equivalent, the function $F=F(t, k, X)$ in explicit form. In this expression, the parameter

$n=\delta^{-2} \tanh ^{2}(k / 2)=\tanh ^{2}(k / 2)\left[\frac{\operatorname{coth}(k / 2)-(k / 2)}{(k / 2)-\tanh (k / 2)}\right]$

depends only on $k$. For $k$ values within the instability interval, this parameter is $0<n<\infty$.

Note that, for any fixed value of $\theta_{0}$ and $t \rightarrow \infty$, it follows from (3.15) that $Y(t) \rightarrow 1$; correspondingly, $\tan [\theta(t) / 2] \rightarrow \delta$. In view of (3.10), $Y=1$ corresponds to a growing normal mode. Below, it is shown that this mode is not an optimal perturbation even at large time values $t \rightarrow \infty$. The value $F$ for a normal-mode $F=F_{n}=e^{2 s(k) t}$ is obtained from (3.16) at $X=1$.

For fixed values of $t, k$, within the interval $0<\theta_{0}<\pi$ $\left(0<X<\delta^{-1}\right)$, function $F(3.16)$ has a single maximum point $\theta_{0}=\theta_{\text {opt }}\left(X=X_{\text {opt }}\right)$, which corresponds to an

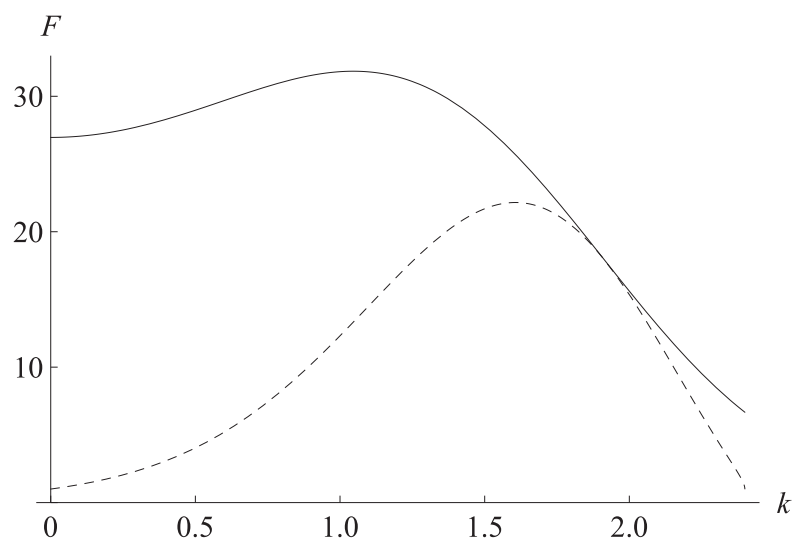

FIG. 6. Dependences of $F_{\text {opt }}$ (solid line) and $F_{n}$ (dashed line) on the wavenumber $k$ at $t=5$.

optimal perturbation (Fig. 5). Differentiating (3.16) with respect to $X$ and equating the derivative to zero, we obtain the quadratic equation

$$
X^{2}-c(t)(n-1) X-n=0 .
$$

Hence, taking into account the variation range of the argument, we obtain the maximum point

$$
\begin{aligned}
X_{\mathrm{opt}} & =\delta^{-1} \tan \left(\theta_{\mathrm{opt}} / 2\right) \\
& =\frac{1}{2}\left[\sqrt{c^{2}(t)(n-1)^{2}+4 n}+c(t)(n-1)\right]
\end{aligned}
$$

and the corresponding maximum value

$F_{\mathrm{opt}}=1+\frac{(n+1) c^{2}(t)}{1-c^{2}(t)}\left[1+\frac{1}{c(t) X_{\mathrm{opt}}}\right], \quad c(t)=\tanh [s(k) t]$.

Equations (3.19) and (3.20) give an analytical solution of the problem of optimal perturbations with zero PV. These new formulas determine the dependence of the optimal phase shift and the maximum of the ratio of energies on the wavenumber and optimization time.

According to (3.19), the phase shift for an optimal perturbation generally depends on the optimization time $t$. The exception is the only wavenumber value $k=$ $k_{1}=1.915$, for which $n=n(k)=1$ and $\tan \left(\theta_{\mathrm{opt}} / 2\right)=$ $\delta=\tan \left(\theta_{n} / 2\right)\left(X_{\mathrm{opt}}=1\right)$. It is easy to check that $n(k)=1$ is reduced to equation $\tanh k=k / 2$ obtained earlier for initial optimal perturbations. Thus, for $k=k_{1}$ and any $t \geq 0$, both optimal and normal modes coincide. For all other values $\tan \left(\theta_{\text {opt }} / 2\right) \neq \tan \left(\theta_{n} / 2\right)$; correspondingly, $F_{\text {opt }}>F_{n}$. Figure 6 shows the dependences of $F_{\text {opt }}$ and $F_{n}$ on the wavenumber $k$ at the fixed time $t=5$. As in the case of initial optimal perturbations, the graphs touch each other at the point $k=k_{1}$. 


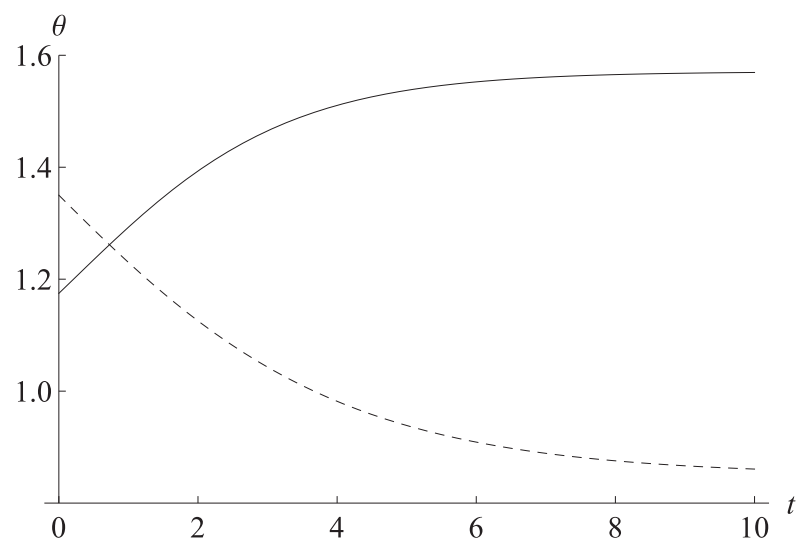

FIG. 7. Time dependences of the phase shift $\theta_{\text {opt }}$ at $k=1.606$ (solid line) and $k=2.2$ (dashed line).

Let us give the asymptotic forms [which follow from (3.19)] for an optimal phase shift at $t \rightarrow 0$ and $t \rightarrow \infty$ $[c(t) \rightarrow 0$ and $c(t) \rightarrow 1$, respectively]

$$
\begin{aligned}
\tan \left(\theta_{\mathrm{opt}} / 2\right) \sim \delta n^{1 / 2}= & \tanh (k / 2), \quad t \rightarrow 0, \\
\tan \left(\theta_{\mathrm{opt}} / 2\right) \sim \delta n= & \tanh ^{2}(k / 2)\left[\frac{\operatorname{coth}(k / 2)-(k / 2)}{(k / 2)-\tanh (k / 2)}\right]^{1 / 2}, \\
& t \rightarrow \infty .
\end{aligned}
$$

In view of (3.10), the first asymptotic form yields the phase shift for an initial optimal perturbation. With increasing $t$, the phase shift increases if $k<k_{1}(n>1)$ and decreases if $k>k_{1}$ (Fig. 7).

The asymptotic forms for the maximum value follow from (3.20):

$$
\begin{array}{ll}
F_{\mathrm{opt}} \sim 1+\frac{k}{\sinh (k)} t, & t \rightarrow 0, \\
F_{\mathrm{opt}}-1 \sim\left(F_{n}-1\right)\left[1+\frac{1}{4} \frac{(n-1)^{2}}{n}\right], & t \rightarrow \infty,
\end{array}
$$

where $F_{n}=\exp [2 s(k) t]$. The first asymptotics may be written in the form $F_{\mathrm{opt}} \sim 1+\gamma_{\mathrm{opt}} t$, where $\gamma_{\mathrm{opt}}=k / \sinh (k)$ corresponds to (3.9) for initial optimal perturbations. The other asymptotics shows that, at $n \neq 1$ and $t \rightarrow \infty$, the ratio $F_{\text {opt }} / F_{n}$ always exceeds one and depends only on the wavenumber. If, at fixed $k$, one draws the graphs of the time dependences of $F_{\text {opt }}$ and $F_{n}$ on a logarithmic scale, they will have the form of two parallel lines located one above the other (Fig. 8).

Note that the first asymptotics (3.23) is also valid for finite $t$ values at $k \rightarrow 0, k_{b}$ when $s(k) \rightarrow 0$. In view of this fact, it follows from (3.23) that $F_{\text {opt }}$ linearly increases with time in the vicinity of the boundaries of the instability interval $0<k<k_{b}$ and exponentially increases

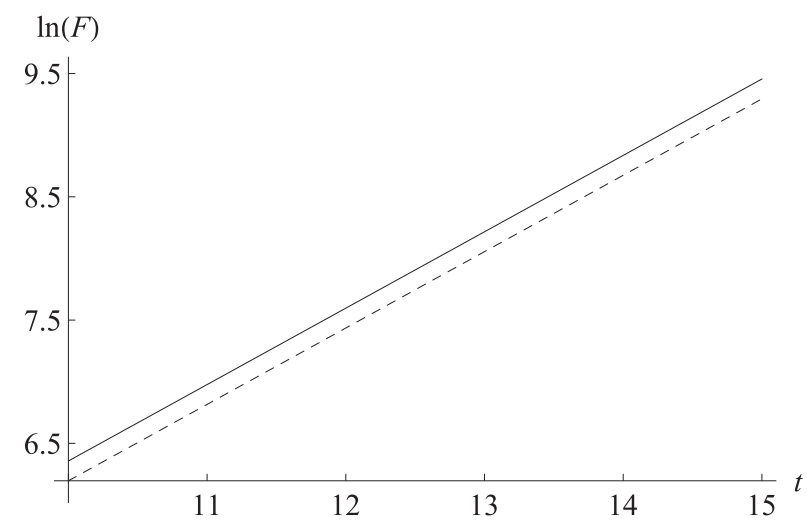

FIG. 8. Time dependences of $\ln \left(F_{\text {opt }}\right)$ (solid line) and $\ln \left(F_{n}\right)$ (dashed line) at $k=1.606$.

with time at any inner point. In this case, for finite $t$ values, $F_{\text {opt }}$ reaches its maximum at some inner point $k=k_{\max }(t)$ of the interval; that is, the preferred value of the wavenumber appears (see Fig. 6). At $t=5$ (optimization time on the order of 2 days), this value $k_{\max } \sim 1.2$.

Before concluding this section, let us note that representation (3.16) of the function $F=F\left(t, k, \theta_{0}\right)$ expressed through the half-angle tangent is used in the given solution of the problem of optimal perturbations. Using the formula $\tan (\theta / 2)=\sin \theta /(1+\cos \theta)$, one can represent (3.16) in terms of basic trigonometric functions:

$$
\begin{aligned}
F= & 1+\left[\frac{c(t)}{1-c^{2}(t)}\right]\left[\frac{k \sinh (k)}{1-\alpha^{2}}\right] \\
& \times\left\{\frac{\sqrt{1-\alpha^{2}} \sin \left(\theta_{0}\right)+c(t)\left[1-\alpha \cos \left(\theta_{0}\right)\right]}{\cosh (k)-\cos \left(\theta_{0}\right)}\right\} .
\end{aligned}
$$

To find extreme points, the trigonometric equation follows from (3.24):

$$
\begin{aligned}
& \left(1-\alpha^{2}\right)^{1 / 2} \cosh (k) \cos \left(\theta_{0}\right)+c(t)[\alpha \cosh (k)-1] \\
& \quad \times \sin \left(\theta_{0}\right)-\left(1-\alpha^{2}\right)^{1 / 2}=0,
\end{aligned}
$$

which (after transition to the half-angle tangent) is equivalent to (3.18). The asymptotics $F \sim 1+\gamma t, t \rightarrow 0$, where the energy growth rate $\gamma$ is determined from (3.8), follows directly from (3.24). This asymptotics is in complete agreement with the asymptotic expansion of energy ratio (3.11).

\section{c. The case of unequal boundary amplitudes}

Above, it was assumed that the optimal perturbations belong to the class of perturbations with equal boundary amplitudes. In the analysis of the problem of optimal perturbations, free from this assumption, we use the complete system of dynamical (2.14a) and (2.14b). 
If we put $a=\rho(t) \cos [\varphi(t)]$ and $b=\rho(t) \sin [\varphi(t)]$, the system can be reduced to equations

$$
\begin{aligned}
d \varphi / d t & =r \sin (\theta) \cos (2 \varphi), \\
d \theta / d t & =-2 r\left[\alpha-\sin ^{-1}(2 \varphi) \cos (\theta)\right],
\end{aligned}
$$

and the equation $d \rho / d t=r \rho \sin \theta \sin 2 \varphi$. By virtue of the nonnegativity of the amplitudes, in this system $0<$ $\varphi<\pi / 2$ and the solution with equal amplitudes corresponds to $\varphi=\pi / 4$.

In view of the fact that the amplitudes are represented in the polar coordinates, the total perturbation energy

$E(t)=\frac{1}{4} \frac{\operatorname{coth}(k)}{k} \rho^{2}(t)\left\{1-\cosh ^{-1}(k) \sin [2 \varphi(t)] \cos \theta(t)\right\}$,

and the energy ratio

$$
F=\frac{E(t)}{E(0)}=\left[\frac{\rho^{2}(t)}{\rho^{2}(0)}\right]\left\{\frac{\cosh (k)-\sin [2 \varphi(t)] \cos [\theta(t)]}{\cosh (k)-\sin \left(2 \varphi_{0}\right) \cos \left(\theta_{0}\right)}\right\} .
$$

First integrals (2.16) yield the ratios

$\frac{\rho^{2}(t)}{\rho^{2}(0)}=\frac{\cos \left(2 \varphi_{0}\right)}{\cos [2 \varphi(t)]}=\frac{\alpha-\sin \left(2 \varphi_{0}\right) \cos \left(\theta_{0}\right)}{\alpha-\sin [2 \varphi(t)] \cos [\theta(t)]}$,

each of which may be used for the transformation of (3.27). Using the first ratio, we obtain

$$
\begin{aligned}
F= & \frac{E(t)}{E(0)}=\left\{\frac{\cos \left(2 \varphi_{0}\right)}{\cos [2 \varphi(t)]}\right\} \\
& \times\left\{\frac{\cosh (k)-\sin [2 \varphi(t)] \cos [\theta(t)]}{\cosh (k)-\sin \left(2 \varphi_{0}\right) \cos \left(\theta_{0}\right)}\right\} .
\end{aligned}
$$

In this expression, $\theta(t), \varphi(t)$ are the solutions of system (3.26), which parametrically depend on wavenumber and initial conditions: $\theta(t)=\theta\left(t, k, \theta_{0}, \varphi_{0}\right), \varphi(t)=\varphi\left(t, k, \theta_{0}, \varphi_{0}\right)$. In view of these dependences, (3.29) determines the function $F=F\left(t, k, \theta_{0}, \varphi_{0}\right)$, which is considered for fixed $t, k$ values. The optimization problem is reduced to finding initial conditions $\theta_{0}, \varphi_{0}$, for which this function takes on its maximum value.

If the optimal perturbation has equal boundary amplitudes, then for arbitrary $t, k, \theta_{0}$ values, the function $F$ reaches its maximum at $\varphi_{0}=\pi / 4$. Let us show that this is so for the wavenumber $k_{s}=k=2.065$, which satisfies the condition $\alpha(k)=0$. In this case, the system of (3.26) is simplified. Thus, ratios (3.28) yield the dependence

$\cos \theta=\chi \tan (2 \varphi), \quad \chi=\tan \left(2 \varphi_{0}\right) \cos \left(\theta_{0}\right)$, in view of which, we can reduce the first equation $[(3.26)]$ to the equation

$$
d \varphi / d t=r \cos (2 \varphi) \sqrt{1-\chi^{2} \tan ^{-2}(2 \varphi)}, \quad \varphi(0)=\varphi_{0} .
$$

Integrating (3.31), after transformations, we obtain the solution in the form

$\cos (2 \varphi)=\frac{\cos \left(2 \varphi_{0}\right) \sqrt{1-p^{2}(t)}}{1+p(t) \sin \left(2 \varphi_{0}\right) \sin \left(\theta_{0}\right)}, \quad p(t)=\tanh (2 r t)$,

where (recall that) $r=\sinh ^{-1}(k)$. Substituting (3.30) and (3.32) into (3.29), we will find the explicit representation for the function $F$ :

$$
F=\frac{1}{\sqrt{1-p^{2}(t)}}\left[1+\frac{d \sin \left(2 \varphi_{0}\right)}{\cosh (k)-\sin \left(2 \varphi_{0}\right) \cos \left(\theta_{0}\right)}\right],
$$

where

$$
d=p(t) \cosh (k) \sin \left(\theta_{0}\right)+\left[1-\sqrt{1-p^{2}(t)}\right] \cos \left(\theta_{0}\right) .
$$

A simple investigation of (3.33) for an extremum shows that it is reached at $\varphi_{0}=\pi / 4$ (i.e., at $a=b$ ). In fact, the equation $\partial F / \partial \varphi_{0}=0$ is reduced to $\cos \left(2 \varphi_{0}\right)$. Substituting $\varphi_{0}=\pi / 4$ into the equation $\partial F / \partial \theta_{0}=0$, we obtain the equation $\cosh (k) \cos \left(\theta_{0}\right)-\tanh (r t) \sin \left(\theta_{0}\right)-1=0$ to find the value of $\theta_{0}=\theta_{\text {opt }}$. Since, at $\alpha=0$, the normal-mode growth rate is $s=\sinh ^{-1}(k)=r$, the last equation is equivalent to (3.25) or, in terms of half-angle tangent, to (3.18).

Similarly, but using more cumbersome expressions, one can show that that $a=b$ for arbitrary $k$ values.

\section{d. Other norms}

Along with the total energy, other quadratic functionals (norms) can be used as a measure of the intensity of the perturbations. Similar to the previous one, for each norm, one can find optimal perturbations. In the existing works, as a rule, the following norms are considered (Fischer 1998; Faulwetter 2006):

$$
\begin{aligned}
& G_{1}=\frac{1}{2}\left\langle\psi_{x}^{2}+\psi_{z}^{2}\right\rangle, \quad G_{2}=\frac{1}{2}\left\langle\psi_{z}^{2}\right\rangle, \quad G_{3}=\frac{1}{2}\left\langle\psi_{x}^{2}\right\rangle, \\
& G_{4}=\left.{\overline{\psi^{2}}}^{x}\right|_{z=0}+\left.\bar{\psi}^{2}\right|_{z=1}, \quad G_{5}=\left.\bar{\psi}_{z}^{2}\right|_{z=0}+\left.{\overline{\psi_{z}^{2}}}^{x}\right|_{z=1} .
\end{aligned}
$$

The first three norms represent, respectively, the total, potential, and kinetic energies. Norms $G_{4}$ and $G_{5}$ are the so-called boundary pressure norm and boundary buoyancy (potential temperature) norm. Note that the 
norm $G_{3}$ (kinetic energy) is equivalent to the norm $L_{2}$ for the streamfunction.

Using expression (2.12) for the streamfunction, each of the norms (3.34) can be represented in the form

$$
G_{i}=n_{i}(k)\left[a^{2}+b^{2}-2 g_{i}(k) a b \cos \theta\right] .
$$

Here, $a=a(t), b=b(t)$, and $\theta=\theta(t)$ is a solution of the system of (2.14), and the functions $n_{i}(k), g_{i}(k)$ are determined taking into account the structure of a particular norm.

Let us first consider perturbations with a maximum norm growth rate at the initial instant of time. Using the first integrals (2.16), for the time derivative $G_{i}$, we obtain

$$
\begin{aligned}
d G_{i} / d t & =4 n_{i}(k) h_{i}(k) a b \sin (\theta), \\
h_{i}(k) & =\left[1-\alpha(k) g_{i}(k)\right] / \sinh (k),
\end{aligned}
$$

where the function $\alpha=\alpha(k)$ is defined by the expression (2.7). Hence, the norm growth rate

$$
\gamma_{i}=\frac{1}{G_{i}} \frac{d G_{i}}{d t}=\frac{4 h_{i}(k) a b \sin (\theta)}{a^{2}+b^{2}-2 g_{i}(k) a b \cos (\theta)},
$$

and for perturbations with equal boundary amplitudes $a=b$,

$$
\gamma_{i}=\frac{1}{G_{i}} \frac{d G_{i}}{d t}=\frac{2 h_{i}(k) \sin (\theta)}{1-g_{i}(k) \cos (\theta)} .
$$

The study (3.37) on the extremum gives the dependence of the parameters of the initial optimal perturbations on the wavenumber $k$ :

$$
\cos \left(\theta_{\mathrm{opt}}\right)=g_{i}(k), \quad \gamma_{\mathrm{opt}}=\gamma\left(k, \theta_{\mathrm{opt}}\right)=\frac{2 h_{i}(k)}{\sqrt{1-g_{i}^{2}(k)}}
$$

According to (3.38), the nature of these dependences is determined by the function $g_{i}(k)$ entering into the expression for the norm (3.35). For the above norms, a direct calculation yields

$$
\begin{aligned}
& g_{1}(k)=\cosh ^{-1}(k), \quad g_{2}(k)=\frac{2[\sinh (k)-k \cosh (k)]}{\sinh (2 k)-2 k}, \\
& g_{3}(k)=\frac{2[\sinh (k)+k \cosh (k)]}{\sinh (2 k)+2 k}, \quad g_{4}(k)=\frac{2 \cosh (k)}{1+\cosh ^{2}(k)}, \\
& g_{5}(k)=0 .
\end{aligned}
$$

Equations (3.38), which correspond to $g_{1}(k)$, reduce to (3.9) for initial optimal perturbations in the energy
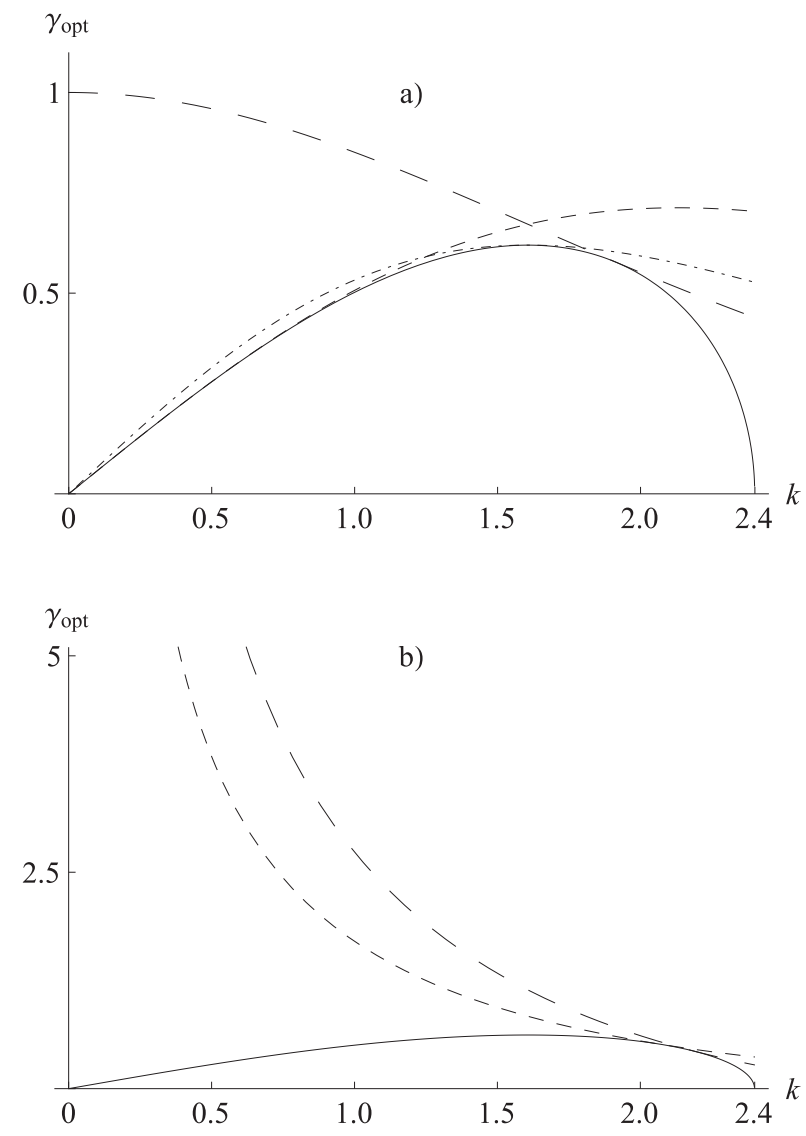

FIG. 9. (a) The graphs of $\gamma_{n}(k)$ (solid line) and $\gamma_{\text {opt }}(k)$ for norms $G_{1}$ (line with long dashes, $G_{3}$ (dashed line), and $G_{4}$ (dash-dotted line). (b) The graphs of $\gamma_{n}(k)$ (solid line) and $\gamma_{\text {opt }}(k)$ for norms $G_{2}$ (line with long dashes) and $G_{5}$ (dashed line).

norm. We note that for the norm $G_{5}$ the optimal phase shift $\theta_{\text {opt }}$ is independent of the wave number and is equal to $\pi / 2$. The corresponding maximum value $\gamma_{\mathrm{opt}}=2 / \sinh (k)$.

For increasing normal modes with $a=b$ and $\cos \theta_{n}=$ $\alpha(k)$, the value $\gamma_{i}$ (3.37) is equal to twice the growth rate $s(k)(2.10)$ in each norm. As the analysis shows, there is only one normal mode, which is the optimal perturbation. According to (3.38), the wavenumber of this mode $k=k_{*_{i}}$ is the root of the equation $s_{i}(k)=$ $\alpha(k)$. We give the values of the root for norms (3.34): $k_{*_{1}}=1.915, k_{*_{2}}=2.190, k_{*_{3}}=0, k_{*_{4}}=k_{m}=1.606$, and $k_{* 5}=k_{s}=2.065$. It is interesting to note that the most unstable normal mode $\left(k=k_{m}\right)$ is the optimal perturbation with respect to the boundary norm $G_{4}$.

Figure 9 shows the graphs of $\gamma_{n}(k)=2 s(k)$ and $\gamma_{\text {opt }}(k)$ for different norms $G_{i}$. For norms $G_{2}$ and $G_{5}, \gamma_{\text {opt }}(k) \rightarrow \infty$ at $k \rightarrow 0$; that is, in the limit of long waves, an infinite growth rate is reached. This is consistent with the results of Faulwetter (2006). Note that the given graphs are tangent at points $k=k *_{i}$. 
As for the total energy, the parameters of the optimal perturbations with the maximum of the ratio $F=$ $G_{i}(t) / G_{i}(0)$ can be found. Taking into account (3.35) and the first integrals (2.16), this ratio can be represented in the form

$$
F=\frac{G_{i}(t)}{G_{i}(0)}=\left[\frac{\alpha(k)-\cos \left(\theta_{0}\right)}{1-g_{i}(k) \cos \left(\theta_{0}\right)}\right]\left\{\frac{1-g_{i}(k) \cos [\theta(t)]}{\alpha-\cos [\theta(t)]}\right\},
$$

similar to (3.13). Taking into account the exact solution (3.15) for the phase shift $\theta(t)$, one can obtain an explicit expression for the function $F=F\left(t, k, \theta_{0}\right)$ and then use the standard analysis for the extremum. This analysis is most simply carried out for the norm $G_{5}$, when $g_{5}(k)=0$. After going to the half-angle tangent, the expression for $F$ reduces to (3.16), where now $n=\delta^{2}=(1+\alpha) /(1-\alpha)$. With this substitution, (3.19) determines the value of the initial optimal phase shift $\theta_{0}$ (for a given instant of time). Accordingly, (3.20) determines the maximum value $F$. The normal mode with $n=1\left[\alpha(k)=0, k=k_{s}\right]$ is the optimal perturbation for any $t>0$.

This brief analysis shows that the parameters of the optimal perturbations depend on the choice of the norm. In the previous sections, we used the energy norm, taking into account the important physical meaning of this characteristic. The equation of energy balance allows us to give a physical interpretation of the baroclinic instability.

\section{Optimal excitation of baroclinic waves by a singular PV distribution}

In the previous sections, we considered free baroclinic waves-perturbations with PV $=0$. Their development is initiated by the initial buoyancy distributions at the boundaries of the atmosphere layer. The excitation of baroclinic waves can also be caused by their interaction with vortex perturbations $(\mathrm{PV} \neq 0)$. In this section, we consider the problems associated with the regimes of optimal excitation. a. Representation of the solution of the general initial-value problem for periodic perturbations: Wave and vortex components

In the dimensionless form, the general solution of the transport equation $\mathrm{PV}[(2.1)]$ is represented in the form $q=q_{i}(x-z t, z)$. Here, $q_{i}(x, z)$ is the initial distribution of PV. The description of the dynamics of perturbations of a flow with a vertical shear, thus, reduces to solving the Poisson equation,

$$
\psi_{x x}+\psi_{z z}=q_{i}(x-z t, z),
$$

with boundary conditions (2.3). The solution of this problem can be represented by the sum of the wave and vortex components

$$
\psi=\psi^{(v)}(x, z, t)+\psi^{(w)}(x, z, t) .
$$

Here, the vortex component $\psi^{(v)}$ is defined as the solution of (4.1) with the conditions $\psi_{z}=0$ for $z=(0,1)$ (conditions of the absence of buoyancy perturbations at the boundaries).

The wave component $\psi^{(w)}$ is defined as the solution of the Laplace equation $\psi_{x x}^{(w)}+\psi_{z z}^{(w)}=0$ with the boundary conditions following from (2.3):

$$
z=0,1: \quad(\partial / \partial t+z \partial / \partial x) \psi_{z}^{(w)}-\psi_{x}^{(w)}=\psi_{x}^{(v)} .
$$

The right-hand side of (4.3) describes the source of generation of baroclinic waves by a vortex component.

We represent the initial distribution of $\mathrm{PV}$ in the form

$$
q_{i}(x, z)=\Phi(z) \exp (i k x),
$$

where the function $\Phi(z)$ describes the vertical distribution structure. For such a distribution, the vortex component $\psi^{(v)}(x, z, t)=\tilde{\psi}(z, t) \exp (i k x)$, where $\tilde{\psi}(z, t)$ is the solution of the boundary value problem

$d^{2} \tilde{\psi} / d^{2} z-k^{2} \tilde{\psi}=\Phi(z) \exp (-i k z t),\left.\quad \tilde{\psi}_{z}\right|_{z=0,1}=0$.

Hence, for the vortex component, we obtain

$$
\begin{aligned}
\psi^{(v)} & =\exp (i k x) \int_{0}^{1} G(z, \xi) \Phi(\xi) \exp (-i k \xi t) d \xi, \\
G(z, \xi) & =-\frac{1}{k \sinh (k)}\left\{\begin{array}{ll}
\cosh (k z) \cosh [k(\xi-1)], & 0<z<\xi, \\
\cosh (k \xi) \cosh [k(z-1)], & \xi<z<1
\end{array} .\right.
\end{aligned}
$$

where $G(z, \xi)$ is the Green's function of the boundary value problem (4.5).
We seek the wave component with the zero distribution PV in the form (2.4). Determining the values of 
$\psi^{(v)}$ at the boundaries from (4.6), after substituting (2.4) into the boundary conditions (4.3), we obtain an inhomogeneous version of the system (2.6)

$$
\begin{aligned}
i d \mathbf{r} / d t & =\mathbf{S r}+\mathbf{F}, \quad \mathbf{F}=\left(f_{1}, f_{2}\right), \\
f_{1} & =r \int_{0}^{1} \cosh (k \xi) \Phi(\xi) e^{-i k \xi t} d \xi, \\
f_{2} & =r \int_{0}^{1} \cosh [k(\xi-1)] \Phi(\xi) e^{-i k \xi t} d \xi,
\end{aligned}
$$

where $r=\sinh ^{-1}(k)$. From the linear system (4.7), we find the complex amplitudes $A(t), B(t)$ of the wave component, and then, according to (4.2), the solution of the problem with given initial amplitudes is determined. Note that instead of the distribution (4.4), we can consider a more general distribution $q_{i}=\Phi(z) \exp [i(k x+\eta)]$, where $\eta$ is the initial phase. In the expressions for the wave and vortex components, we must replace $k x$ by $k x+\eta$.

\section{b. Singular distribution of $P V$}

We will consider the case of the initial singular distribution PV:

$$
\Phi(z)=a_{v} \delta(z-h),
$$

where $\delta(z)$ is a delta function and $a_{v}$ is a real amplitude parameter. Taking into account (4.6), for this distribution the vortex component,

$$
\psi^{(v)}=a_{v} G(z, h) e^{i\left(k x-\omega_{v} t\right)}, \quad \omega_{v}=k h .
$$

Equation (4.10) (real part) describes a vortex wave with frequency $\omega_{v}$ and a phase velocity $c_{x}=h$ equal to the flow velocity at the level $z=h$. In plasma physics, such a wave is often called the Van Kampen-Case wave or a continuous mode (Van Kampen 1955; Timofeev 1971; Kalashnik and Chkhetiani 2017). Another name is KRW proposed by Heifetz and Methven (2005). A characteristic feature of the structure of this wave is the jump of the vertical derivative (the discontinuity of the buoyancy distribution) at the source level. Signs of perturbation of buoyancy on both sides of the jump are opposite; that is, the perturbation has a dipole structure.

The propagation of a vortex wave (4.10) leads to the excitation of baroclinic waves (the wave component of the solution). The description of the excitation process can be obtained based on the solution of system (4.7) with right-hand sides

$$
\begin{aligned}
& f_{1}=a_{v} r \cosh (k h) \exp \left[i \theta_{v}(t)\right], \\
& f_{2}=a_{v} r \cosh [k(h-1)] \exp \left[i \theta_{v}(t)\right],
\end{aligned}
$$

where the phase $\theta_{v}(t)=-\omega_{v} t$ is introduced (in the general case, $\left.\theta_{v}(t)=\eta-\omega_{v} t\right)$. Another way is to convert
(4.7) to a system of equations for real amplitudes and phases. This is done using the real part of (4.11) and the representation for the amplitudes (2.11). The corresponding system, called the three-wave system, was first formulated by Dirren and Davies (2004), and subsequently analyzed by de Vries et al. (2009). The system includes two equations for the amplitudes,

$$
\begin{aligned}
d a / d t & =r b \sin (\theta)+a_{v} r \cosh (k h) \sin \left(\theta_{v}-\theta_{2}\right), \\
\theta & =\theta_{1}-\theta_{2}, \\
d b / d t & =r a \sin (\theta)+a_{v} r \cosh [k(h-1)] \sin \left(\theta_{v}-\theta_{1}\right),
\end{aligned}
$$

and two equations for the phases $\theta_{1}, \theta_{2}$. As shown by Dirren and Davies (2004), if at the initial moment of time, the condition $a=b$ and condition

$$
\theta_{v}-0.5\left(\theta_{1}+\theta_{2}\right)=\pi / 2
$$

are satisfied, then for $h=0.5$ (a vortex source in the center of the layer), a synchronous excitation regime is realized. This regime is described by a system of two equations:

$$
\begin{aligned}
& d a / d t=r a \sin (\theta)+a_{v} r \cosh (k / 2) \cos (\theta / 2), \\
& d \theta / d t=-2 r[\alpha-\cos (\theta)]-2\left(a_{v} / a\right) r \cosh (k / 2) \sin (\theta / 2) .
\end{aligned}
$$

For $a_{v}$, the system (4.14) reduces to the system (2.14a) and (2.14b).

Based on the numerical solution (4.12) and (4.14), Dirren and Davies (2004) investigated various scenarios of baroclinic wave excitation. Note that the phase values in this work differ from the above (another normalization).

\section{c. Energy balance equation and initial optimal excitation}

The energy balance equation gives a physical interpretation of the process of excitation of baroclinic waves. We define the density and the total-energy $E^{(w)}$ of the wave component by (3.1), where $\psi=\psi^{(w)}$. Since for the wave component $q=0$, horizontal averaging of (3.2) leads to (3.3) for the horizontally averaged density $\bar{\varepsilon}^{x}$. Integrating the last equation with respect to $z$ and using the boundary conditions (4.3) multiplied by $\psi^{(w)}$, we obtain the energy balance equation for the wave component in the form

$$
\begin{aligned}
\frac{d E^{(w)}}{d t} & =\left.{\overline{\psi_{x}^{(w)} \psi_{z}^{(w)}}}^{x}\right|_{z=1}+J, \\
J & =\left.{\overline{\psi^{(w)} \psi_{x}^{(v)}}}^{x}\right|_{z=1}-{\left.\overline{\psi^{(w)} \psi_{x}^{(v)}}\right|_{z=0} .}^{x} .
\end{aligned}
$$

Unlike (3.4), this equation contains an additional source $J$ describing the interaction between the wave and vortex components at the boundaries. 
Equation (4.15) allows us to formulate the problem of optimal excitation of baroclinic waves at the initial instant of time. We shall call optimal the excitation, for which the energy growth rate of the wave component $\gamma=\left[1 / E^{(w)}\right] d E^{(w)} / d t$ assumes the maximum value.

The determination of the parameters of the optimal excitation reduces to an analysis of the explicit expression for $\gamma$. Taking into account the representation (2.12), the total energy of the wave component and the energy flux at the upper boundary are given by (3.6) and (3.5), where the phase shift is $\theta=\theta_{1}-\theta_{2}$. Using (2.12) and (4.10), a direct calculation of the source $J$ (4.15) yields

$$
\begin{aligned}
J= & 0.5 a_{v} r k^{-1}\left\{a \sinh (k h) \sin \left(\theta_{v}-\theta_{1}\right)\right. \\
& \left.+b \sinh [k(1-h)] \sin \left(\theta_{v}-\theta_{2}\right)\right\} .
\end{aligned}
$$

For the energy growth rate of the wave component, we thus obtain

$\gamma=\frac{1}{E^{(w)}} \frac{d E^{(w)}}{d t}=\frac{2 k}{\cosh (k)} \frac{a b \sin (\theta)+2 r^{-1} J}{a^{2}+b^{2}-2 a b \cosh ^{-1}(k) \cos (\theta)}$,

which is (for a fixed value of the wavenumber) a function of seven parameters $a, b, a_{v}, \theta_{1}, \theta_{2}, \theta_{v}$, and $h$. In some cases, the analysis of this function on an extremum is carried out analytically.

We restrict ourselves to the case of equal boundary amplitudes $a=b$ and $h=0.5$. Equation (4.16) for a vortex source is then transformed to the form

$J=\frac{a_{v} a \sinh (k / 2)}{k \sinh (k)} \sin (\varphi) \cos (\theta / 2), \quad \varphi=\theta_{v}-0.5\left(\theta_{1}+\theta_{2}\right)$.

It follows immediately from (4.18) that the maximum of $J$ is attained at $\varphi=\pi / 2$, that is, when the synchronous growth condition (4.13) is satisfied. If this condition is satisfied, then taking into account (4.17), the energy growth rate of the wave component

$$
\gamma=\frac{1}{E^{(w)}} \frac{d E^{(w)}}{d t}=k \frac{\sin (\theta)+\lambda \rho \sin (\theta / 2)}{\cosh (k)-\cos (\theta)} .
$$

Here, $\rho=2 \sinh (k / 2) / k$ is denoted, and the amplitude parameter $\lambda=a_{v} / a$ is introduced. For $\lambda=0$ (the absence of a vortex source), (4.19) for $\gamma=\gamma(k, \lambda, \theta)$ reduces to $(3.8)$.

Using (4.19), it is not difficult to verify that the derivative $d \gamma / d \theta$ has different signs at the ends of the interval $0<$ $\theta<\pi$. Hence, it follows that, for any value of $\lambda \geq 0$ on this interval, there is a maximum point $\theta=\theta_{\text {opt }}(k, \lambda)$, which corresponds to the maximum value $\gamma=\gamma_{\mathrm{opt}}(k, \lambda)$. The

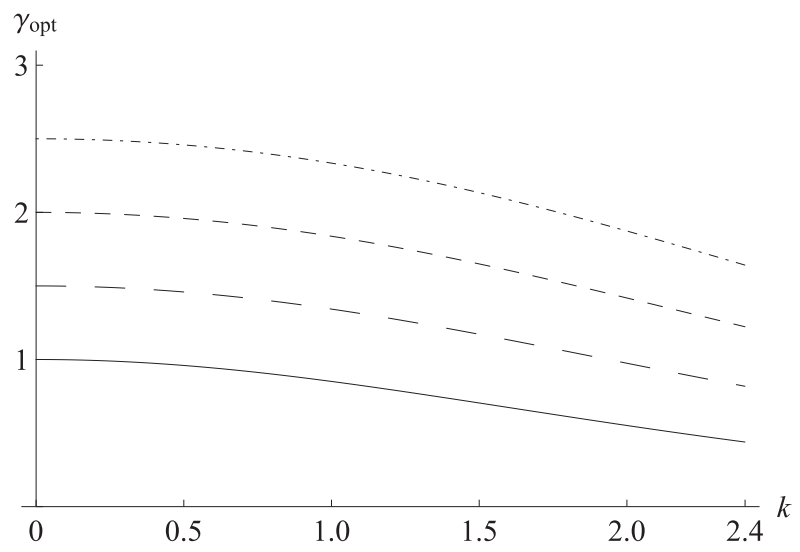

FIG. 10. Dependences of $\gamma_{\text {opt }}$ on the wavenumber $k$ for $\lambda=0$ (solid line), $\lambda=1$ (line with long dashes), $\lambda=2$ (dashed line), and $\lambda=3$ (dash-dotted line).

equation $d \gamma / d \theta=0$ for finding the maximum point reduces to a cubic equation with respect to $\sin (\theta / 2)$, and its solution is cumbersome. The dependences $\gamma_{\mathrm{opt}}(k)$ for various values of $\lambda$ obtained numerically are shown in Fig. 10. We give also the characteristic asymptotics valid for finite values of $k>0$ :

$$
\begin{gathered}
\cos \theta_{\text {opt }}=\cosh ^{-1}(k)+\lambda \frac{\tanh ^{2} k \cosh ^{2}(k / 2)}{k \sqrt{\cosh (k)}}, \quad \lambda \ll 1, \\
\sin \left(\theta_{\text {opt }} / 2\right)=2 \lambda^{-1} k \sinh (k / 2) /[\cosh (k)+3], \quad \lambda \gg 1 .
\end{gathered}
$$

The corresponding maximum values of $\gamma$,

$$
\begin{array}{ll}
\gamma_{\mathrm{opt}}=k \sinh ^{-1}(k)+\lambda \cosh ^{-1 / 2}(k), & \lambda \ll 1, \\
\gamma_{\mathrm{opt}}=\lambda \sinh ^{-1}(k / 2), & \lambda \gg 1 .
\end{array}
$$

Asymptotics (4.21) and Fig. 10 show that in the presence of the vortex component, the energy growth rate $\gamma_{\text {opt }}$ increases, and the characteristic time of energy growth $\tau_{\mathrm{opt}}=1 / \gamma_{\mathrm{opt}}$ decreases. For the wavenumber $k=1.6$ and the values $\lambda$ shown in Fig. 11, this dimensional time is $\tau_{\text {opt }}=41.4 \mathrm{~h}(\lambda=0), \tau_{\text {opt }}=21.4 \mathrm{~h}(\lambda=1), \tau_{\text {opt }}=15.4 \mathrm{~h}$ $(\lambda=2)$, and $\tau_{\text {opt }}=11.5 \mathrm{~h}(\lambda=3)$. Here, it is important to emphasize that the growth of $\gamma_{\mathrm{opt}}$ refers to the initial instant of time. Since the amplitude of the vortex component $a_{v}=$ const, and the amplitude of the wave component $a$ exponentially increases, over time, the wave component will play the dominant role in the dynamics. The vortex component plays only the role of a catalyst, leading to the excitation of baroclinic waves.

It follows from the presented analysis that in the optimal excitation regime the synchronous growth condition (4.13) and condition $a=b$ must be fulfilled. The phase shift 

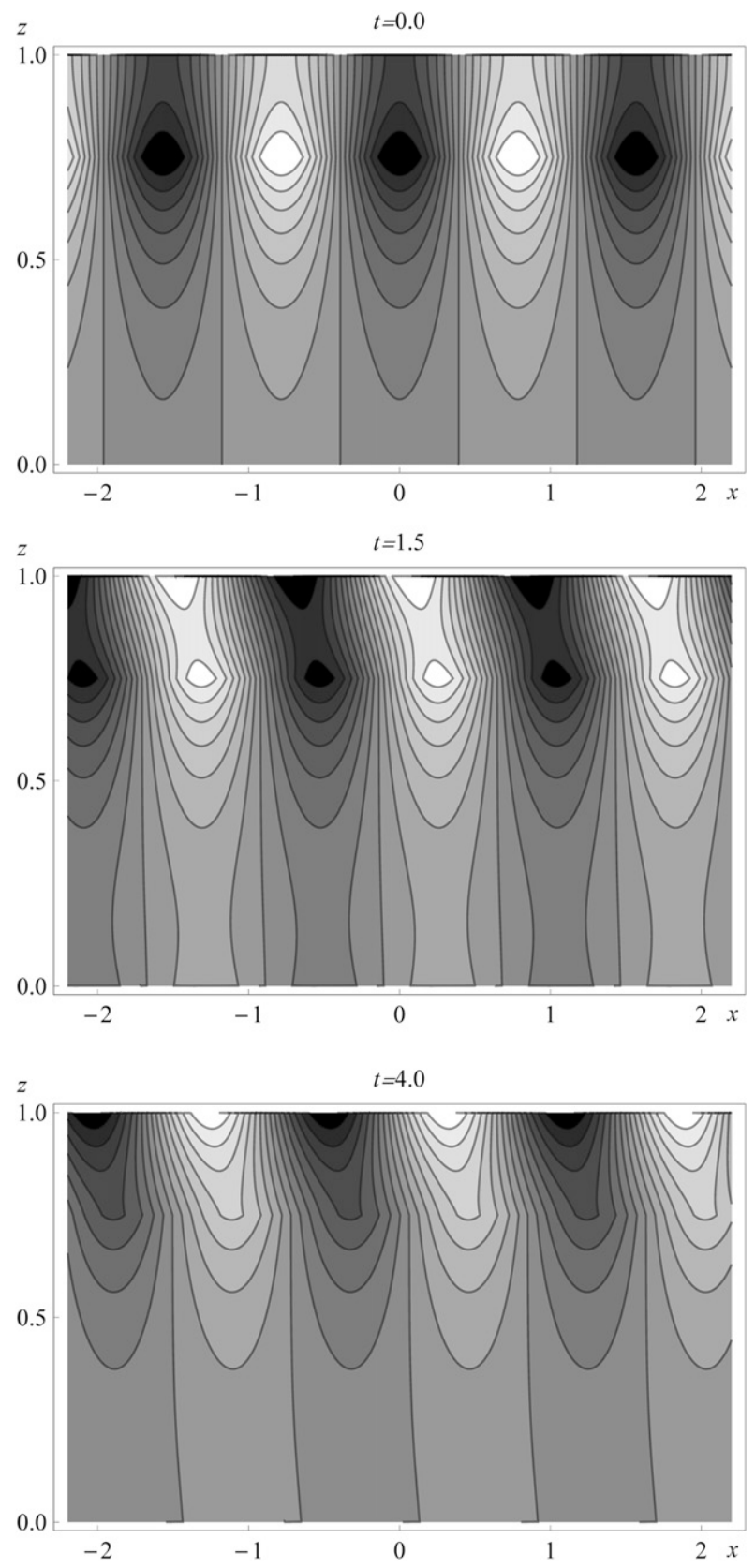

FIG. 11. Grayscale plots of the isolines of the pressure field $\psi$ for (top to bottom) three instants of time $t=0, t=1$, and $t=4$ under resonance conditions.

$\theta$ between the buoyancy distributions at the boundaries should be equal to the optimal value $\theta=\theta_{\text {opt }}(k, \lambda)$.

We note that the exact expressions for the parameters of the optimal excitation can be obtained in the case of the norm $G=G_{5}$ (boundary buoyancy norm). Taking into account (2.12), for this norm, $G=0.5\left(a^{2}+b^{2}\right)$ and $G_{t}=a a_{t}+b b_{t}$. Using (4.12), for the norm growth rate at $a=b$ and $h=0.5$, we obtain

$$
\begin{aligned}
\gamma=G^{-1} d G / d t= & 2 r \sin \theta \\
& +\lambda \rho r\left[\sin \left(\theta_{v}-\theta_{2}\right)+\sin \left(\theta_{v}-\theta_{1}\right)\right],
\end{aligned}
$$

where now $\rho=\cosh (k / 2)$. If condition (4.13) is satisfied, this expression is transformed to the form

$$
\gamma=2 r[\sin (\theta)+\lambda \rho \cos (\theta / 2)] .
$$

On the interval $0<\theta<\pi$, the maximum point (4.23) is found from the equation $1-2 \sin ^{2}(\theta / 2)-\sin (\theta / 2)=0$. For an optimal phase shift, we obtain

$\sin \left(\theta_{\mathrm{opt}} / 2\right)=1 /\left(\varepsilon+\sqrt{2+\varepsilon^{2}}\right), \quad \varepsilon=\lambda \rho / 4$.

If $\lambda=0$, then $\theta_{\text {opt }}=\pi / 2$. For $\lambda \ll 1$, it follows from (4.24) that $\theta_{\text {opt }} \sim \pi / 2-\varepsilon \sqrt{2}$. The maximum value of the norm growth rate is given by the exact expression

$$
\gamma_{\mathrm{opt}}=2 r\left[2 \sin \left(\theta_{\mathrm{opt}} / 2\right)+\lambda \rho\right]\left[1-\sin ^{2}\left(\theta_{\mathrm{opt}} / 2\right)\right]^{1 / 2} .
$$

We also give characteristic asymptotics:

$$
\begin{array}{ll}
\gamma_{\text {opt }}=2 \sinh ^{-1}(k)+\lambda \sqrt{2} / 2 \sinh (k / 2), & \lambda \ll 1, \\
\gamma_{\text {opt }}=\lambda / \sinh (k / 2), & \lambda \gg 1 .
\end{array}
$$

As for the energy norm, with increasing amplitude of the vortex component, the growth rate $\gamma_{\text {opt }}$ increases. We note that since the vortex component at the boundaries of the atmosphere layer is zero, the value of the boundary norm $G_{5}$ is determined only by the wave component. The considered examples show that the problem of the initial optimal excitation of baroclinic waves is mathematically posed correctly; that is, it has a unique solution. A more general problem of optimal excitation of baroclinic waves with a maximum ratio of finite and initial energies (norms) requires the solution of the systems of (4.14) or (4.12). This question is beyond the scope of this article.

\section{d. Resonant excitation of neutral waves}

Everywhere above, we considered the interval of wavenumbers $0<k<k_{b}$ for which there exist exponentially increasing normal modes. The propagation of a vortex wave (4.10) with a wavenumber $k>k_{b}$ can lead to the resonance excitation of neutral baroclinic waves, in which their amplitude grows linearly (in the absence of instability). This effect was first described by Thorncroft and Hoskins (1990) and subsequently studied in Chang (1992), Jenkner and Ehrendorfer (2006), de Vries and Opsteegh (2007), and Kalashnik (2015). Below is the frequency interpretation of the resonance, and the connection 
with the problem of optimal perturbations is discussed. For the sake of brevity, we shall use a complex form of solutions, everywhere assuming the selection of the real part.

The process of excitation of neutral waves $\left(k>k_{b}\right)$ can be conveniently carried out using the analytical solution of system (4.7). We shall consider this system with the initial condition $\mathbf{r}(0)=0$ corresponding to the absence of the wave component at the initial instant of time. Determining from (4.7) the amplitudes $A(t), B(t)$ (the method of variation of arbitrary constants) and substituting in (2.4), for the streamfunction of the wave component, we obtain the expression (2.9), where $C_{1,2}=C_{1,2}(t)$,

$$
\begin{aligned}
& C_{1,2}(t)=i \int_{0}^{t} \tilde{f}_{1,2}(\tau) e^{i \omega_{1,2} \tau} d \tau, \\
& \tilde{f}_{1,2}(\tau)=\frac{f_{2}(1 \mp \delta)-f_{1}(1 \pm \delta)}{4 \delta} .
\end{aligned}
$$

For the singular distribution (4.9), the functions $f_{1}, f_{2}$ are defined by (4.11). Wherein

$$
\begin{aligned}
C_{1,2}(t) & =a_{v} \mu_{1,2} \frac{e^{i\left(\omega_{1,2}-\omega_{v}\right) t}-1}{\omega_{1,2}-\omega_{v}}, \\
\mu_{1,2} & =-\frac{\sinh (k)(h-0.5)}{4 \delta \cosh (0.5 k)} \mp \frac{\cosh [k(h-0.5)]}{4 \sinh (0.5 k)},
\end{aligned}
$$

and the streamfunction of the wave component

$$
\begin{aligned}
\psi^{(w)}= & a_{v} \mu_{1} F_{1}(z) \frac{e^{i\left(k x-\omega_{1} t\right)}-e^{i\left(k x-\omega_{v} t\right)}}{\omega_{v}-\omega_{1}} \\
& -a_{v} \mu_{2} F_{2}(z) \frac{e^{i\left(k x-\omega_{2} t\right)}-e^{i\left(k x-\omega_{v} t\right)}}{\omega_{v}-\omega_{2}} .
\end{aligned}
$$

According to (4.27), the propagation of the vortex wave (4.10) leads to the excitation of three neutral baroclinic waves with frequencies $\omega_{1}, \omega_{2}$ (respectively, upper and lower ERW) and frequency $\omega_{v}$.

The validity of the solution (4.27) is violated in resonance cases, when $\omega_{v}=\omega_{1}$ or $\omega_{v}=\omega_{2}$. The resonant wavenumber $k=k_{r}$, at which the frequencies coincide, depends on the position of the source $h$. If $h>0.5$, then $k_{r}$ is found from the equation $\omega_{v}(k)=\omega_{2}(k)$. If $h<0.5$, then $k_{r}$ is the root of the equation $\omega_{v}(k)=\omega_{1}(k)$. Taking into account (2.7) for frequencies, the corresponding roots always exist.

Passing to the limit $\omega_{1} \rightarrow \omega_{v}$ in (4.27), for the first resonance case, we obtain the solution

$$
\begin{aligned}
\psi^{(w)}= & i a_{v} \mu_{1} t F_{1}(z) e^{i\left(k x-\omega_{v} t\right)} \\
& -a_{v} \mu_{2} F_{2}(z) \frac{e^{i\left(k x-\omega_{2} t\right)}-e^{i\left(k x-\omega_{v} t\right)}}{\omega_{v}-\omega_{2}}
\end{aligned}
$$

linearly growing with time. A similar growing solution corresponds to the second case of $\omega_{2} \rightarrow \omega_{v}$. Thus, resonant excitation is realized when the frequency of the vortex wave (4.10) coincides with one of the frequencies of baroclinic waves (ERWs).

Figure 11 give the grayscale plots of the isolines $\psi=$ $\psi^{(w)}+\psi^{(v)}$ (pressure field) for three instants of time and the value $h=3 / 4$. This value corresponds to the resonant wavenumber $k=k_{r}=4.005$ (wavelength of order $1500 \mathrm{~km}$ ). A vortex wave with this wavenumber excites a baroclinic wave (ERW) localized at the upper boundary. The amplitude of the ERW increases linearly with time and is compared with the amplitude of the vortex wave in a time on the order of 2 days. The baroclinic wave localized at the lower boundary is excited at $h<1 / 2$.

The energy balance equation [(4.15)] allows one to interpret the resonance in terms of the phase shift between the boundary distributions $\psi^{(w)}$ and $\psi_{x}^{(v)}$, which appear in the expression for the source $J$. We represent the solution (4.28) in the form $\psi^{(w)}=\psi_{1}^{(w)}+\psi_{2}^{(w)}$, where $\psi_{1}^{(w)}$ corresponds to a linearly increasing part with time. With allowance for $\mu_{1}<00$, for this part and the distribution of $\psi_{x}^{(v)}$ on the lower boundary, we have (real form)

$$
\begin{aligned}
& \left.\psi_{1}^{(w)}\right|_{z=0}=a_{v}\left|\mu_{1}\right| F_{1}(0) t \sin (\vartheta), \\
& \left.\psi_{x}^{(v)}\right|_{z=0}=a_{v} r \cosh [k(h-1)] \sin (\vartheta),
\end{aligned}
$$

where $\vartheta=k_{x}-\omega_{v} t$. As can be seen, the distributions (4.29) have equal phases. Similarly, the equality of phases at the upper boundary is checked. With this equality in mind, a direct calculation of the source $J=J_{1}$ (4.15), corresponding to $\psi^{(w)}=\psi_{1}^{(w)}$, yields

$$
J=J_{1}=a_{v}^{2} \frac{\sinh (2 k h)}{2 \sinh (k)} t,
$$

which increases linearly with time. It is easy to verify that the contribution of the term $\psi_{2}^{(w)}$ to the source $J$ is a periodic function of time that vanishes when averaged over a time period. According to (4.15), in the presence of the source (4.30), the energy of the wave component increases according to a quadratic law. This resonant growth occurs under the conditions of coincidence of the frequencies and equal phases of the wave and vortex components at the boundaries.

The presented analysis motivates the following formulation of the problem of optimal perturbations in the regime of neutral waves $k>k_{b}$. We shall call the initial vortex perturbation (4.10) optimal if it leads to an unbounded growth of the energy of the excited wave component. The answer to the question of optimal perturbation is unambiguous. The vortex perturbation (4.10) with the wavenumber $k$ and the $h$ level is optimal if one of the conditions 

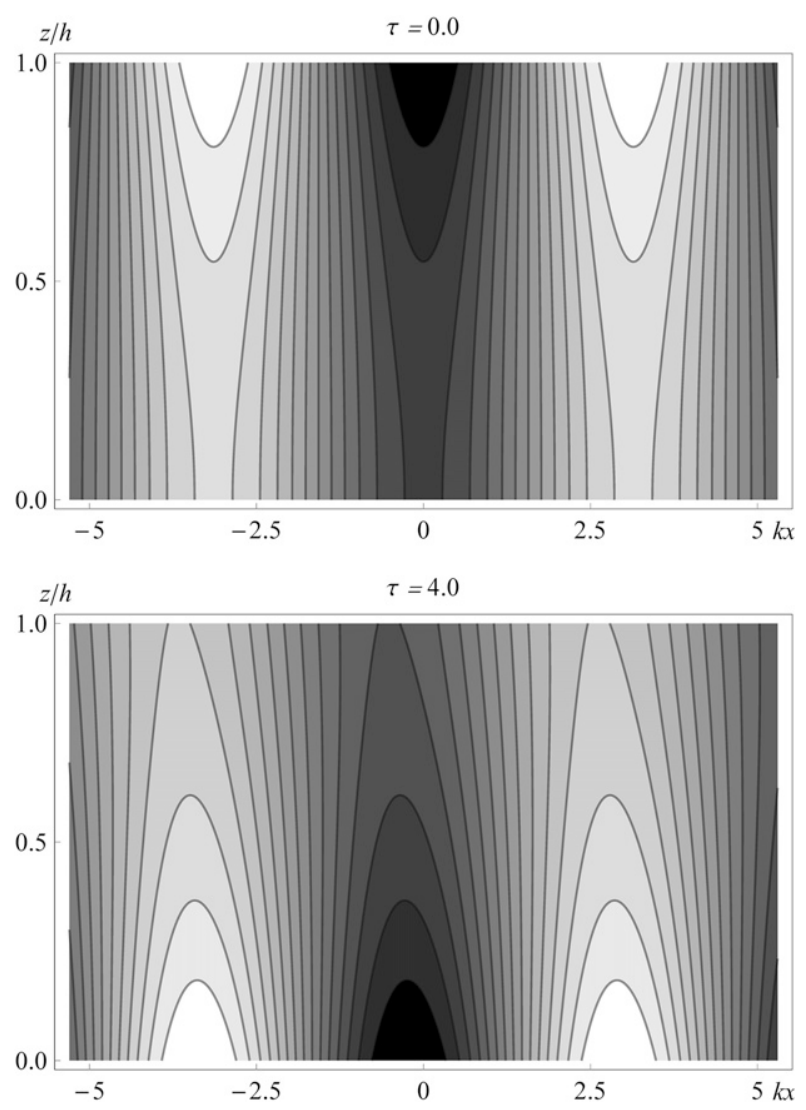

FIG. 12. Grayscale plots of the isolines of the pressure field $\psi$ for (top and bottom) two instants of time, respectively, under quasiresonance conditions.

$\omega_{1}(k)=k h$ or $\omega_{2}(k)=k h$ is satisfied. For all other relations between the parameters, the energy of the wave component oscillates (the energy input into the system is compensated for by a flow of energy in another interval). This formulation of the problem is attractive in that its solution does not depend on the amplitude of the vortex perturbation.

We note that the resonance effect does not exhaust all the interesting features of the interaction of the vortex and wave components. Thus, in the case of a discontinuous function $\Phi(z)$ describing the vertical distribution structure of PV (4.4), quasi-resonant excitation of baroclinic waves with logarithmic growth of their amplitudes can be realized (Kalashnik 2015). This question is briefly discussed in the appendix. Grayscale plots of the isolines of the pressure field $\psi$ under quasi-resonance conditions are presented in Fig. 12 (for two instants of time).

\section{Conclusions}

Extremal problems associated with nonnormal differential operators-non-self-adjoint operators without the property of orthogonality of eigenfunctions-are considered in the theory of optimal perturbations. In this theory, the conventional eigenfunction expansion of perturbations is replaced by the expansion in terms of the so-called singular vectors. In the Eady model, existing numerical methods of finding optimal perturbations are based mainly on these mathematical notions. In this paper, a direct approach, which makes it possible to find analytically the parameters of optimal perturbations without using the indicated notions, is proposed. This approach is based on explicit expressions for the energy growth rate and ratios between final and initial energies, which follow from the representation of the perturbations with zero PV through the superposition of ERWs. The corresponding expressions are the functions of the parameters of initial perturbations, and determining optimal parameters is reduced to studying conventionally these functions for an extremum. The proposed approach can be used in the analysis of a general initial-value problem, whose solution is represented by the sum of the wave and vortex components, respectively, with zero and nonzero PV.

On the basis of the proposed approach, three problems are solved in the paper: 1) the problem of optimal perturbations with a maximum of the energy growth rate $(\mathrm{PV}=0), 2)$ the problem of optimal perturbations with a maximum of the ratio of the final and initial energies $(P V=0)$, and 3$)$ the problem of the optimal excitation ERWs regime by a singular vortex wave or KRW $(P V \neq 0)$.

The main new results in solving the first two problems consist in obtaining analytical expressions for the optimal phase shift and the maximum energy ratio (energy growth rate) that determine their dependence on the wavenumber and optimization time. These expressions provide basic information about optimal perturbations with zero PV. In the course of solving the first two problems, we also compare the parameters of the optimal perturbations with the parameters of the growing normal modes in different norms. It is established that in the energy norm there exists only one exponentially increasing normal mode, which is the optimal perturbation. The wavenumber of this mode is different from the wavenumber of the normal mode with the growth rate maximum.

The new result of the third problem, first of all, is its formulation - the problem of the optimal regime of excitation of the wave component of the solution by a vortex component. In this case, the optimal regime is understood as the regime with the maximum energy growth rate of the wave component (ERWs) at the initial instant of time. Since it is the ERWs that describe the development of cyclones, this formulation seems 
physically justified. As shown in the paper, the optimal regime is realized only with a special phase shift of two ERWs, which depends on the wavenumber and the amplitude of the initial vortex perturbation (KRW amplitude). The asymptotic expressions for this shift and the corresponding maximum of the energy growth rate are obtained. It is shown that a vortex disturbance leads to an increase in the energy growth rate of ERWs, which is in full agreement with the numerical calculations of Dirren and Davies (2004).

In conclusion, we note that the approach proposed in this paper is transferred to other problems of the theory of hydrodynamic stability. These include the classical problem of the stability of a free shear layer (Heifetz et al. 1999; Heifetz and Methven 2005), the problem of the stability of shear flows with a free surface (Mallios and Bakas 2017), and the problem of the stability of a geostrophic flow with a vertical and horizontal shear (Barcilon and Bishop 1998; Kalashnik 2009). Using the perturbation representation of the sum ERWs, for all these problems, explicit expressions for the energy functionals can be obtained. The parameters of the optimal perturbations are found from the analysis of these expressions for the extremum.

Acknowledgments. This work was supported by the Russian Science Foundation (Project 14-17-00806). Analysis of optimal perturbations with zero PV (section 3) was supported by the Russian Foundation for Basic Research (Project 18-05-00831).

\section{APPENDIX}

\section{Quasi-Resonance Excitation of Baroclinic Waves with Logarithmic Growth of Their Amplitudes}

To illustrate the process of excitation, we consider a model of a semibounded atmosphere $z \geq 0$. The dynamics of two-dimensional perturbations of a shear flow is described by (2.1) with the boundary condition (2.2) at $z=0$. Since the model does not have a characteristic vertical scale, we use dimensional variables everywhere.

As in section 4, (2.1) reduces to

$$
\psi_{x x}+\delta^{-2} \psi_{z z}=q_{i}(x-\Lambda z t, z), \quad \delta=N / f,
$$

where $q_{i}(x, z)$ is a given initial distribution of PV. We represent the streamfunction in the form $\psi=$ $\psi^{(v)}(x, z, t)+\psi^{(w)}(x, z, t)$, where the vortex component $\psi^{(v)}$ is defined as the solution of (A.1) with the condition $\psi_{z}=0$ at $z=0$ and the boundedness condition for $z \rightarrow \infty$. For the distribution of $q_{i}(x, z)=\Phi(z) \exp ^{(i k x)}$, this component

$$
\begin{aligned}
\psi^{(v)} & =\delta^{2} \exp (i k x) \int_{0}^{\infty} G(z, \xi) \Phi(\xi) \exp (-i k \Lambda \xi t) d \xi, \\
G(z, \xi) & =-\frac{1}{\mu}\left\{\begin{array}{ll}
e^{-\mu \xi} \cosh (\mu z), & 0<z<\xi, \\
e^{-\mu z} \cosh (\mu \xi), & \xi<z<\infty .
\end{array}, \mu=\delta k,\right.
\end{aligned}
$$

where $G(z, \xi)$ is the Green's function of the boundary value problem analogous to (4.5),

$$
d^{2} \tilde{\psi} / d^{2} z-\mu^{2} \tilde{\psi}=g(z), \quad \tilde{\psi}_{z}(0)=0,\left.\quad \tilde{\psi}\right|_{z=\infty}=O(1) .
$$

We define the wave component as the solution of the homogeneous (A.1) with the boundary condition following from (2.1):

$$
z=0: \quad \psi_{z t}^{(w)}-\Lambda \psi_{x}^{(w)}=\Lambda \psi_{x}^{(v)} .
$$

We seek this component in the form

$$
\psi^{(w)}=-\mu^{-1} A(t) \exp (-\mu z) \exp (i k x),
$$

where $A(t)$ is the complex amplitude. Determining from (A.2) the value of $\psi^{(v)}$ on the boundary and substituting (A.4) into condition (A.3), to determine the amplitude, we obtain

$d A / d t+i \omega A=-F(t)$,

$$
F(t)=i \omega \int_{0}^{\infty} \exp (-\mu z) \Phi(z) \exp (-i \Lambda k z t) d z
$$

which we will consider with the initial condition $A(0)=$ 0 . Here, $\omega=\Lambda \delta^{-1}=\Lambda f / N$ is the frequency of baroclinic waves, which are always neutral in the model of a semibounded atmosphere.

The representation of the solution (A.5) in the form $A(t)=C(t) e^{i \omega t}$ leads to

$$
\begin{aligned}
& \psi^{(w)}=\mu^{-1} C(t) e^{-\mu z} \operatorname{expi}(k x-\omega t), \\
& C(t)=\int_{0}^{t} F(\tau) e^{i \omega \tau} d \tau .
\end{aligned}
$$

The finding of the wave component, therefore, reduces to the calculation of the integrals entering into (A.5) and (A.6). An analysis of these integrals shows that the character of the time behavior of the wave component depends on the structure $\Phi(z)$ of the vertical distribution of PV. In the case of a singular distribution of PV (4.9), a resonance with a linear increase of the amplitude of the baroclinic waves is realized. If the distribution $\Phi(z)$ contains discontinuities, quasi-resonant excitation with logarithmic time growth can be observed. For smooth 
distributions of $\Phi(z)$, waves of finite amplitude are always excited. The quasi-resonant excitation is analyzed below, which, apparently, was not considered in the literature.

As a distribution with one point of discontinuity, we take

$$
\begin{array}{ll}
\Phi(z)=0, & 0<z<h, \\
\Phi(z)=a_{v} \exp (-z / h), & z>h .
\end{array}
$$

For this distribution, a direct calculation of the integral $F(t)($ A.5) yields

$$
F(t)=i a_{v} \frac{\omega h e^{-(1+d)}}{1+d+i \omega_{v} t} e^{-i \omega_{v} t}, \quad d=\frac{\omega_{v}}{\omega} .
$$

Here, $\omega$ is the frequency of the baroclinic wave, and $\omega_{v}=k \Lambda h$ is the frequency associated with the discontinuity point and is analogous to the frequency of the vortex wave for the singular distribution of PV. Let us consider the case of coincidence of the frequencies $\omega=\omega_{v}$ (quasi resonance), which is realized if $k \delta h=1$. In this case, we find for the function $C(t)$, (A.6)

$$
\begin{aligned}
C(t)= & \operatorname{Im} \int_{0}^{t} \frac{d t}{2+i \omega t}=\frac{m}{2 \omega}\left\{\operatorname{In}\left[1+(\omega t / 2)^{2}\right]\right. \\
& +2 i \arctan (\omega t / 2)\}
\end{aligned}
$$

where $m=a_{v} \omega h e^{-2}$. Substituting (A.8) into (A.6), for the streamfunction of the excited wave component, we obtain (real form)

$$
\begin{aligned}
\psi^{(w)}= & a_{v}(\delta h / e)^{2} e^{-z / h}\left[C_{1}(t) \cos (k x-\omega t)\right. \\
& \left.-C_{2}(t) \sin (k x-\omega t)\right], \\
C_{1}(t)= & 0.5 \operatorname{In}\left[1+(\omega t / 2)^{2}\right], \quad C_{2}(t)=\arctan (\omega t / 2),
\end{aligned}
$$

which increases logarithmically for a long time. The vortex component (A.2) decays according to the law $t^{-1}$ (in contrast to the case of the singular distribution). Note that under quasi-resonance conditions, the buoyancy perturbation $\psi_{z}^{(w)}$ at the boundary $z=0$ is proportional to $h$ (distance to the point of discontinuity). Thus, the more $h$, the more intense the disturbance at the boundary.

Figure 12 give the grayscale plot of the isolines $\psi=\psi^{(w)}+\psi^{(v)}$ (pressure field) for two instants of the dimensionless time $\tau=\omega t=0$ and $\tau=\omega t=4$. The area $z<h$ below the break point of $\Phi(z)$ is shown. At the initial time, only the vortex component exists. Under quasi-resonance conditions, this component excites a baroclinic wave near the boundary. For $h=3 \mathrm{~km}$ and $\delta=10^{2}$, the length of the resonant wave $2 \pi \delta h$ is on the order of $1800 \mathrm{~km}$.
The behavior with logarithmic time growth also occurs in the case of general distributions of the $\Phi(z)$ with one or several points of discontinuity. This is shown using known asymptotic representations for the integrals $F(t)$ (A.5) at $k \rightarrow \infty$. Similar behavior takes place within the framework of the Eady model with two solid boundaries.

\section{REFERENCES}

Barcilon, A., and C. H. Bishop, 1998: Nonmodal development of baroclinic waves undergoing horizontal shear deformation. J. Atmos. Sci., 55, 3583-3597, https://doi.org/ 10.1175/1520-0469(1998)055<3583:NDOBWU>2.0.CO;2.

Borges, M. D., and D. L. Hartmann, 1992: Barotropic instability and optimal perturbations of observed nonzonal flows. J. Atmos. Sci., 49, 335-354, https://doi.org/10.1175/1520-0469(1992)049<0335: BIAOPO $>2.0 . \mathrm{CO} ; 2$.

Chang, E. K., 1992: Resonating neutral modes of the Eady model. J. Atmos. Sci., 49, 2452-2463, https://doi.org/10.1175/15200469(1992)049<2452:RNMOTE $>2.0 . C O ; 2$.

Cushman-Roisin, B., and J.-M. Beckers, 2011: Introduction to Geophysical Fluid Dynamics: Physical and Numerical Aspects. Vol. 101. Academic Press, 875 pp.

Davies, H., and C. Bishop, 1994: Eady edge waves and rapid development. J. Atmos. Sci., 51, 1930-1946, https://doi.org/10.1175/ 1520-0469(1994)051<1930:EEWARD > 2.0.CO;2.

de Vries, H., and J. D. Opsteegh, 2007: Resonance in optimal perturbation evolution. Part I: Two-layer Eady model. J. Atmos. Sci., 64, 673-694, https://doi.org/10.1175/JAS3867.1.

_ J. Methven, T. H. A. Frame, and B. J. Hoskins, 2009: An interpretation of baroclinic initial value problems: Results for simple basic states with nonzero interior PV gradients. J. Atmos. Sci., 66, 864-882, https://doi.org/10.1175/2008JAS2774.1.

Diaconescu, E. P., and R. Laprise, 2012: Singular vectors in atmospheric sciences: A review. Earth-Sci. Rev., 113, 161-175, https://doi.org/10.1016/j.earscirev.2012.05.005.

Dirren, S., and H. C. Davies, 2004: Combined dynamics of boundary and interior perturbations in the Eady setting. J. Atmos. Sci., 61, 1549-1565, https://doi.org/10.1175/15200469(2004)061<1549:CDOBAI > 2.0.CO;2.

Eady, E. T., 1949: Long waves and cyclone waves. Tellus, 1 (3), 33 52, https://doi.org/10.3402/tellusa.v1i3.8507.

Farrell, B. F., 1982: The initial growth of disturbances in a baroclinic flow. J. Atmos. Sci., 39, 1663-1686, https://doi.org/ 10.1175/1520-0469(1982)039<1663:TIGODI > 2.0.CO;2.

_- 1984: Modal and non-modal baroclinic waves. J. Atmos. Sci., 41, 668-673, https://doi.org/10.1175/1520-0469(1984) 041<0668:MANMBW>2.0.CO;2.

_- 1988: Optimal excitation of neutral Rossby waves. J. Atmos. Sci., 45, 163-172, https://doi.org/10.1175/1520-0469(1988)045<0163: OEONRW $>2.0 . \mathrm{CO} ; 2$.

- 1989: Optimal excitation of baroclinic waves. J. Atmos. Sci., 46, 1193-1206, https://doi.org/10.1175/1520-0469(1989) 046<1193:OEOBW>2.0.CO;2.

__ , and P. J. Ioannou, 1996: Generalized stability theory. Part I: Autonomous operators. J. Atmos. Sci., 53, 2025-2040, https://doi.org/10.1175/1520-0469(1996)053<2025:GSTPIA> 2.0.CO;2.

Faulwetter, R., 2006: Optimal growth of Eady edge waves. Meteor. Z., 15, 423-437, https://doi.org/10.1127/0941-2948/2006/0142. 
Fischer, C., 1998: Linear amplification and error growth in the 2D Eady problem with uniform potential vorticity. J. Atmos. Sci., 55, 3363-3380, https://doi.org/10.1175/1520-0469(1998)055<3363: LAAEGI $>2.0 . \mathrm{CO} ; 2$

Heifetz, E., and J. Methven, 2005: Relating optimal growth to counterpropagating Rossby waves in shear instability. Phys. Fluids, 17, 064107, https://doi.org/10.1063/1.1937064.

_ C. Bishop, and P. Alpert, 1999: Counter-propagating Rossby waves in the barotropic Rayleigh model of shear instability. Quart. J. Roy. Meteor. Soc., 125, 2835-2853, https://doi.org/ 10.1002/qj.49712556004.

— - — B. Hoskins, and J. Methven, 2004a: The counterpropagating Rossby-wave perspective on baroclinic instability. I: Mathematical basis. Quart. J. Roy. Meteor. Soc. 130, 211-231, https://doi.org/10.1002/qj.200413059610.

_ propagating Rossby-wave perspective on baroclinic instability. II: Application to the Charney model. Quart. J. Roy. Meteor. Soc., 130, 233-258, https://doi.org/10.1256/qj.02.185.

Jenkner, J., and M. Ehrendorfer, 2006: Resonant continuum modes in the Eady model with rigid lid. J. Atmos. Sci., 63, 765-773, https://doi.org/10.1175/JAS3649.1.

Kalashnik, M. V., 2009: Linear dynamics of Eady waves in the presence of horizontal shear. Izv. Atmos. Oceanic Phys., 45, 714-722, https://doi.org/10.1134/S0001433809060048.

- 2015: Resonant and quasi-resonant excitation of baroclinic waves in the Eady model. Izv. Atmos. Oceanic Phys., 51, 576584, https://doi.org/10.1134/S0001433815060080.

_ , and O. Chkhetiani, 2017: Generation of gravity waves by singular potential vorticity disturbances in shear flows. J. Atmos. Sci., 74, 293-308, https://doi.org/10.1175/JAS-D-16-0134.1.

Leutbecher, M., 2007: On the representation of initial uncertainties with multiple sets of singular vectors optimized for different criteria. Quart. J. Roy. Meteor. Soc., 133, 2045-2056, https:// doi.org/10.1002/qj.174.

Lindzen, R. A., 1990: Dynamics in Atmospheric Physics. Cambridge University Press, 310 pp., https://doi.org/10.1017/ cbo9780511608285.
Mak, M., 2011: Atmospheric Dynamics. Cambridge University Press, 486 pp., https://doi.org/10.1017/cbo9780511762031.

Mallios, C., and N. A. Bakas, 2017: Generalized stability of a shear flow with a free surface with respect to three-dimensional perturbations. Phys. Rev. Fluids, 2, 023901, https://doi.org/ 10.1103/PhysRevFluids.2.023901.

McWilliams, J. C., 2011: Fundamentals of Geophysical Fluid Dynamics. Cambridge University Press, 272 pp.

Morgan, M. C., 2001: A potential vorticity and wave activity diagnosis of optimal perturbation evolution. J. Atmos. Sci., 58, 2518-2544, https://doi.org/10.1175/1520-0469(2001)058<2518: APVAWA $>2.0 . \mathrm{CO} ; 2$.

— , and C.-C. Chen, 2002: Diagnosis of optimal perturbation evolution in the Eady model. J. Atmos. Sci., 59, 169-185, https://doi.org/ 10.1175/1520-0469(2002)059<0169:DOOPEI > 2.0.CO;2.

Mukougawa, H., and T. Ikeda, 1994: Optimal excitation of baroclinic waves in the Eady model. J. Meteor. Soc. Japan, 72, 499513, https://doi.org/10.2151/jmsj1965.72.4.499.

Pedlosky, J., 1987: Geophysical Fluid Dynamics. Springer-Verlag, 710 pp., https://doi.org/10.1007/978-1-4612-4650-3.

Pierrehumbert, R., and K. Swanson, 1995: Baroclinic instability. Annu. Rev. Fluid Mech., 27, 419-467, https://doi.org/10.1146/ annurev.fl.27.010195.002223.

Schmid, P. J., and D. S. Henningson, 2012: Stability and Transition in Shear Flows. Vol. 142. Springer Science and Business Media, 558 pp., https://doi.org/10.1007/978-1-4613-0185-1.

Thorncroft, C. D., and B. J. Hoskins, 1990: Frontal cyclogenesis. J. Atmos. Sci., 47, 2317-2336, https://doi.org/10.1175/15200469(1990)047<2317:FC > 2.0.CO;2.

Timofeev, A. V., 1971: Oscillations of inhomogeneous flows of plasma and liquids. Phys.-Usp., 13, 632-646, https://doi.org/ 10.1070/PU1971v013n05ABEH004220.

Trefethen, L. N., A. Trefethen, S. C. Reddy, and T. A. Driscoll, 1993: Hydrodynamic stability without eigenvalues. Science, 261, 578-584, https://doi.org/10.1126/science.261.5121.578.

Van Kampen, N. G., 1955: On the theory of stationary waves in plasmas. Physica, 21, 949-963, https://doi.org/10.1016/S00318914(55)93068-8. 